\title{
Evaluation of a New Wideband Slot Array for MIMO Performance Enhancement in Indoor WLANs
}

\author{
Jorge R. Costa, Senior Member, IEEE, Eduardo B. Lima, Carla R. Medeiros, and \\ Carlos A. Fernandes, Senior Member, IEEE
}

\begin{abstract}
A new wideband compact slot antenna array for indoor WLAN access points (AP) is described, covering several wireless communication services from 2.4 to $4.8 \mathrm{GHz}$, that is especially designed to enhance MIMO system capacity. The array topology provides both spatial and polarization diversity. Despite very close packing of the array elements, these exhibit very low mutual coupling and low cross-polarization, greatly favoring MIMO diversity gain. A detailed MIMO performance comparison is conducted against a common array of patches in indoor environment, based both on simulation and indoor measurements: the new antenna shows a clear improvement in terms of channel capacity.
\end{abstract}

Index Terms-Printed wideband antenna, tapered slot antenna, multiple input multiple output (MIMO) array, wireless local area network (WLAN), spatial and polarization diversity.

\section{INTRODUCTION}

$\mathbf{T}$ HE benefits from using multiple input and multiple output antennas (MIMO) in wireless communications have been widely discussed in the literature. Spatial diversity improves system reliability by decreasing the sensitivity to fading (diversity gain) without requiring additional bandwidth, unlike the case of frequency or time diversity. Spatial multiplexing can be implemented as an alternative to spatial diversity. In spatial multiplexing each $N_{t}$ transmitter antenna sends an independent data stream, unlike the case of spatial diversity where each antenna sends correlated data (e.g., Alamouti code [1]). With spatial multiplexing the spectral efficiency can be increased by a factor $\min \left(N_{t}, N_{r}\right)$ [2].

Both for spatial diversity and for spatial multiplexing strategies, it is required that the channels between the multiple transmitting $(\mathrm{Tx})$ and receiving $(\mathrm{Rx})$ antennas are uncorrelated to maximize data throughput and for successful data stream decoding. Correlation may result from poor multipath richness of the scenario and from mutual coupling between antenna array elements [3]. Therefore, one important design specification for MIMO antenna arrays is to minimize inter-element coupling.

Manuscript received March 19, 2010; revised August 20, 2010; accepted September 02, 2010. Date of publication January 31, 2011; date of current version April 06, 2011. This work was supported in part by the European Union under project FP7 ICT-2007-216715 (NewCom++).

J. R. Costa is with the Instituto de Telecomunicações, IST, 1049-001 Lisboa, Portugal and also with the Departamento de Ciências e Tecnologias da Informação, ISCTE-Lisbon University Institute, 1649-026 Lisboa, Portugal (e-mail: Jorge.Costa@1x.it.pt).

E. B. Lima, C. R. Medeiros, and C. A. Fernandes are with Instituto de Telecomunicações, IST, 1049-001 Lisboa, Portugal .

Color versions of one or more of the figures in this paper are available online at http://ieeexplore.ieee.org.

Digital Object Identifier 10.1109/TAP.2011.2109685
Polarization diversity was found to be as significant as spatial diversity at improving diversity gain, and hence MIMO system capacity [4]. Therefore, MIMO array elements should also present pure and frequency stable polarization to obtain full benefit from polarization diversity.

The present study aims to present and evaluate a $2 \times 2$ printed array antenna intended to enhance MIMO performance in indoor access points (fixed terminal), with the following cumulative characteristics: low inter-element coupling, pure linear polarization from each array element, good front-to-back ratio for wall mounting, high radiation efficiency, and stability of these characteristics over a large bandwidth to accommodate multiple wireless standards such as WiFi, LTE, WiMAX and part of the UWB band. Furthermore, the four array elements are to be arranged in a compact layout with space and polarization diversity.

The proposed array elements are based upon the linear polarized crossed exponentially tapered slot (XETS) antenna developed by the authors for ultrawideband (UWB) systems [5], [6] and modified in [7] to include a back cavity to change the inherently bidirectional radiation pattern into a unidirectional one. This new configuration is referred as cavity back crossed exponentially tapered slot (CXETS) antenna and presents $67 \%$ bandwidth. The antenna copes with all the specifications listed above and confirmed MIMO improved performance when compared by simulation and measurement to a reference printed patch array with the same element layout and diversity polarization scheme.

To the authors' knowledge, no other antenna reported in the literature copes simultaneously with all the above specifications, which makes the CXETS array a novel alternative for MIMO fixed terminal. Many MIMO arrays were proposed in the literature, but in all cases they match only part of the above requirements [8]-[13], and clearly no solution cumulatively presents comparable characteristics.

This paper is organized as follows. The new CXETS array and the array of patches that is used for comparison are both presented in Section II. Section III describes both the MIMO setup for channel transfer matrix measurements and the performance evaluation of several MIMO configurations with different number of array elements based upon channel capacity estimation. Numerical and experimental results are given and discussed throughout. Conclusions are drawn in Section IV.

\section{ARRAY DESCRIPTION}

Both the XETS and rectangular patch arrays were designed using the transient solver of CST Microwave Studio ${ }^{\mathrm{TM}}$, based on Finite Integration Method [14]. To ensure fair performance 


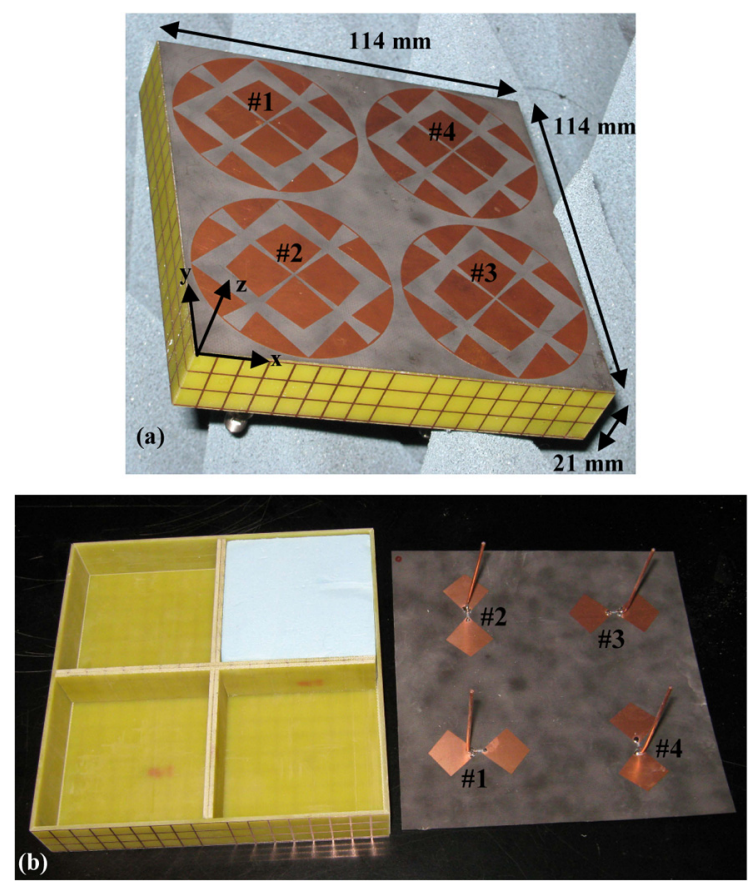

Fig. 1. (a) Photograph of the assembled CXETS array prototype, showing the antenna top face; (b) Disassembled antenna showing the FR4 cavity back and the feeding coaxial cables soldered on the XETS back petals.

comparison between both arrays, they were designed to have similar aperture area.

\section{A. CXETS Array}

The crossed exponentially tapered slot (XETS) antenna configuration was developed by the authors for UWB systems [5], [6]. It is compact, with stable radiation pattern, low cross-polarization and low mutual coupling when packed in a closed array configuration. However, the radiation pattern is bidirectional. To produce a unidirectional radiation pattern, a single antenna was redesigned to include a grid back cavity [7]. This new configuration is onwards referred as the CXETS.

The XETS elements are printed on both sides of DUROID 5880 substrate, $\varepsilon_{r}=2.2, \tan (\delta)=0.0009$ and thickness $h_{5880}=10 \mathrm{mil}=0.254 \mathrm{~mm}$. The top face of the four radiating elements is shown in Fig. 1(a) and the back face, with the feeding coaxial cables, is shown in Fig. 1(b). These are small diameter EZ-47 semi-rigid coaxial cables (1.19 mm diameter), soldered between two replicas of opposing petals from the front face of each XETS, Fig. 1(b). The RF signal is capacitively coupled to the corresponding front petals of each antenna. The fed petals define the E-plane of each XETS. It was verified that common-mode currents resulting from the unbalanced feeding are low in this assembly and do not perturb significantly the expected balanced antenna performance. It is seen in Fig. 1(b) that adjacent elements have orthogonal orientation, so, orthogonal polarization.

The meshed cavity walls are printed on FR4 substrate, $\varepsilon_{r}=$ $4.9, \tan (\delta)=0.025$ and thickness $h_{\mathrm{FR} 4}=1.6 \mathrm{~mm}$. The mesh is uniform, with $6 \mathrm{~mm}$ step. This is a compromise value found through CST simulation that allows retaining a reasonable part of the original antenna bandwidth (now 2.4 to $4.8 \mathrm{GHz}$ ) and at
TABLE I

CXETS ANTENNA PARAmeter VALUes In MiLlimeters

\begin{tabular}{|c|c|c|c|c|c|c|}
\hline$D_{s}$ & $L_{\text {out }}$ & $L_{\text {in }}$ & $w_{s}$ & $L$ & $w_{0}$ & $C_{0}$ \\
\hline 54 & 35.7 & 27.9 & 9 & 53 & 0.72 & 12.7 \\
\hline
\end{tabular}

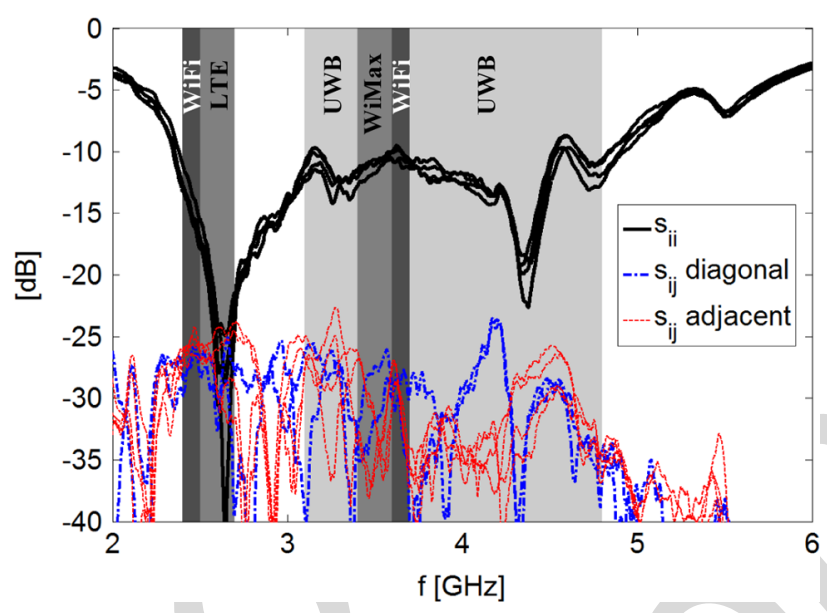

Fig. 2. Measured amplitude of the CXETS array scattering matrix elements.

the same time enables reaching better than $10 \mathrm{~dB}$ front-to-back ratio (f/b). Each array element has its own cavity; the two walls separating each cavity can be observed in Fig. 1(b). The metallic mesh is also printed on the metallic walls. In the assembled antenna, the cavities are filled with low density Styrofoam $\left(\varepsilon_{r} \approx\right.$ $1.05)$ to provide physical support to the thin 10 mils DUROID substrate. The Styrofoam can be observed in one of the cavities of the disassembled antenna in Fig. 1(b).

The remaining parameter values are indicated Table I (the same parameter naming is used as in [5]). The overall size of the array (Fig. 1(a)) is $114 \times 114 \times 21 \mathrm{~mm}^{3}\left(0.91 \lambda_{\text {inf }} \times 0.91 \lambda_{\text {inf }} \times\right.$ $0.17 \lambda_{\text {inf }}$ ) where $\lambda_{\text {inf }}$ is the wavelength at $2.4 \mathrm{GHz}$, the lower operating frequency. Smaller MIMO antenna assemblies have been reported for handheld communication devices, but none of those antennas offer 1:2 bandwidth. The proposed antenna is intended specifically for WLAN access points (fixed terminal).

Fig. 2 presents the measured scattering matrix of the CXETS array. The input reflection coefficient of all elements is below $-10 \mathrm{~dB}$ across the interval from 2.4 to $4.8 \mathrm{GHz}$. This covers WiFi, LTE, WiMax and the lower channels of UWB systems. The corresponding assigned bands are indicated in Fig. 2. It is seen from Fig. 2 that mutual coupling between array elements is mostly below $-25 \mathrm{~dB}$ across the entire antenna bandwidth. It is noted that the original XETS antenna presents very low coupling (about $-70 \mathrm{~dB}$ ) between adjacent cross-polarized array elements [5]. It is the addition of the back cavity that increases mutual coupling to $-25 \mathrm{~dB}$, but this value is still attractive for the present application.

It is known that a N-port antenna efficiency is better characterized by the total active reflection coefficient (TARC) [13], [15] than by the usual inspection of the $\mathbf{S}$-matrix elements. TARC is the square root of the ratio between total reflected power at the array ports and total power fed to the ports. It is calculated by applying a unity magnitude voltage with random phase at each port of the array. In this way, the reflected power translates different combinations of the excitation signals, which is more 


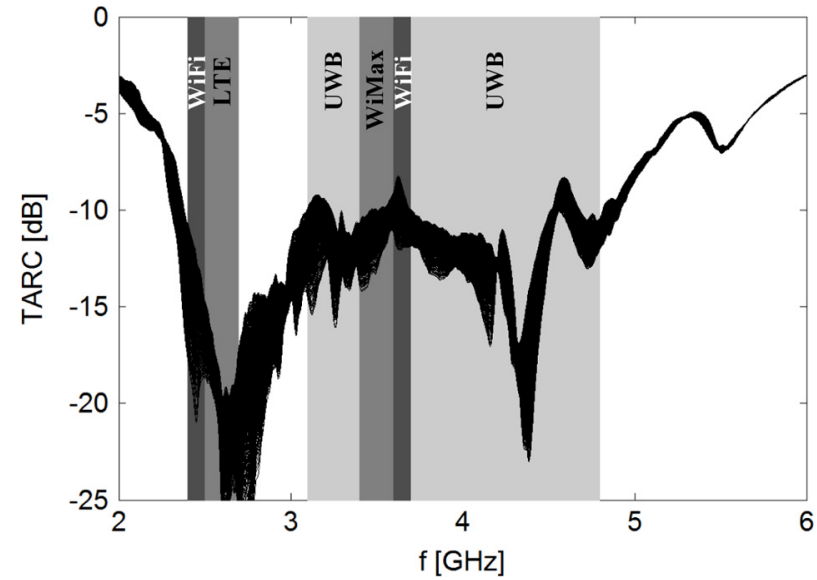

Fig. 3. Measured TARC for the CXETS array prototype.
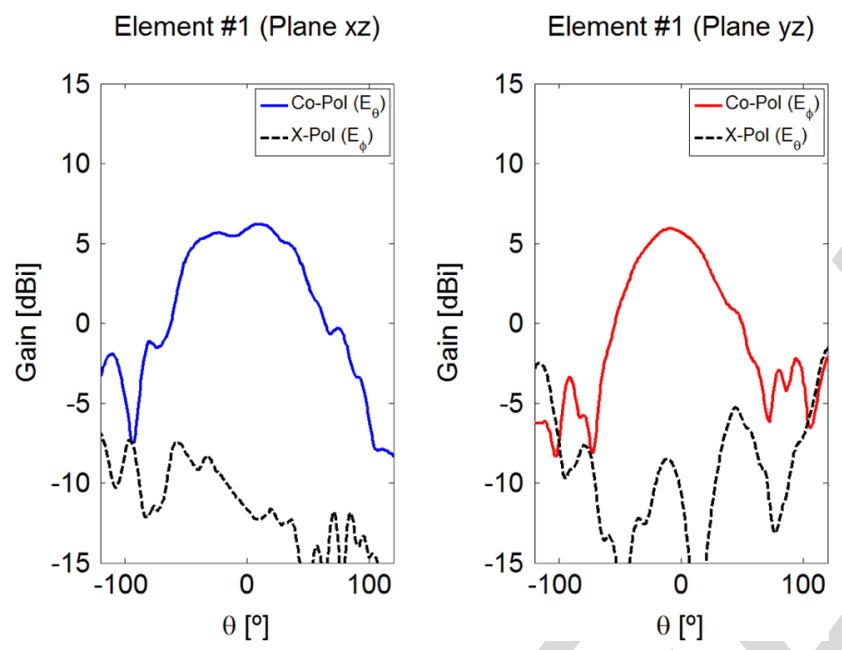

Fig. 4. Measured radiation pattern of the first element of the CXETS array at $2.6 \mathrm{GHz}$.

appropriate to describe the real conditions of a MIMO array operation than the usual single port excitation strategy. It accounts for both coupling and random signal combining between each port signal [13].

The measured scattering matrix of the CXETS was used to compute the antenna TARC. The results for different combinations of the input signals are presented in Fig. 3. Since the CXETS elements present low mutual coupling, TARC level is almost always below $-10 \mathrm{~dB}$ across the bandwidth.

The measured main planes radiation pattern of array element \#1 is presented in Fig. 4, with the other CXETS terminated by matched loads. Vertical scale refers to antenna gain. Cross-polarization level is about $-15 \mathrm{~dB}$. The other three array elements present similar radiation patterns, apart from plane interchange for the $90^{\circ}$ rotated antennas.

The measured front-to-back ratio across the bandwidth varies between 11 and $20 \mathrm{~dB}$ while gain ranges from 5.5 to $8 \mathrm{dBi}$. Cross-polarization improves with frequency from $-15 \mathrm{~dB}$ at 2.4 $\mathrm{GHz}$ to about $-20 \mathrm{~dB}$ at $4.8 \mathrm{GHz}$. CST simulations indicate that the array radiation efficiency is always above $80 \%$.
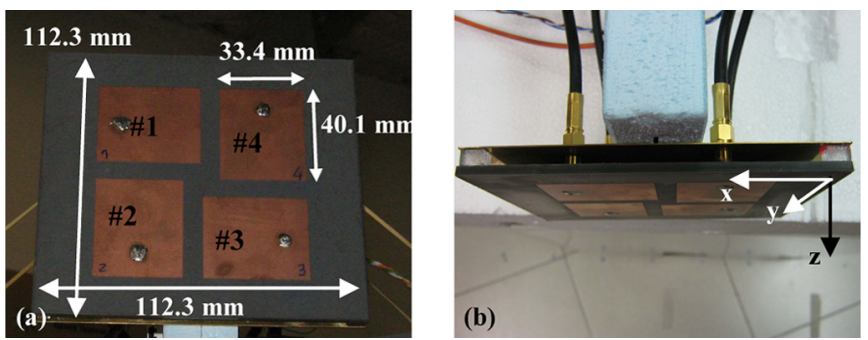

Fig. 5. (a) Photograph of the rectangular patch array prototype; (b) photograph of the air subtract and coaxial feeding.

\section{B. Rectangular Patch Array}

The rectangular patch array prototype is printed on DUROID $5880, \varepsilon_{r}=2.2, \tan (\delta)=0.0009$, thickness $h_{5880}=125 \mathrm{mil}=$ $3.175 \mathrm{~mm}$, see Fig. 5. It was designed to operate in the WiMax or LTE band from 2.5 to $2.7 \mathrm{GHz}$. The desired bandwidth was achieved through the use of an air gap between the dielectric layer and the ground plane $h_{\text {air }}=5 \mathrm{~mm}$. Of course it is not possible to approach the wide CXETS bandwidth with a conventional patch antenna. Patch dimensions $W_{P} \times L_{P}$ are 33.4 $\mathrm{mm} \times 40.1 \mathrm{~mm}\left(0.27 \lambda_{\text {inf }} \times 0.32 \lambda_{\text {inf }}\right)$. Each patch is probe fed by an EZ-141 coaxial cable (3.58 mm diameter). The outer conductor of the feeding coaxial cable is soldered to the ground plane and extended up to the dielectric; this arrangement reduces the excitation of surface waves that can propagate along the air gap and therefore lowers mutual coupling bet $\overline{\overline{\bar{V}}}$ elements as will be seen next. Patches are rotated by $90^{\circ}$ degrees in relation to the adjacent one, Fig. 5. Therefore, the polarization from the patches in one diagonal of the array is orthogonal with respect to the polarization from the other two patches. The ground plane extends by $15.9 \mathrm{~mm}$ from the edges of the patches. With $7.3 \mathrm{~mm}$ gap between adjacent elements, the overall array size is $112.3 \mathrm{~mm} \times 112.3 \mathrm{~mm}\left(0.9 \lambda_{\text {inf }} \times 0.9 \lambda_{\text {inf }}\right)$.

Fig. 6 presents the measured radiation pattern at $2.6 \mathrm{GHz}$ from array patch \#1, with the other patches terminated by matched loads. Vertical scale refers to antenna gain. Cross polarization is high as expected. Very similar radiation patterns were measured for the other three array elements, apart from plane interchange for orthogonal patches.

Fig. 7 presents the measured magnitude of the array scattering matrix elements. Grey shading represents the WiMax or LTE bands. Coupling between adjacent patches reaches $-13.5 \mathrm{~dB}$ within the WiMax or LTE band while coupling between diagonal patches is almost constant across the band at $-15 \mathrm{~dB}$ level. This is an improvement with respect to the usual configuration where the outer conductor of the feeding coaxial cable is terminated at the ground plane. In fact, CST simulations indicate mutual coupling level of $-10 \mathrm{~dB}$ in this case.

Fig. 8 presents TARC curves computed from the measured S-matrix, for random phase and unit amplitude at the excitation ports. It can be observed that the worst value within the WiMax or LTE bands is about $-5 \mathrm{~dB}$. The large dispersion of the TARC curves denotes a strong coupling between antenna elements, unlike the CXETS. 

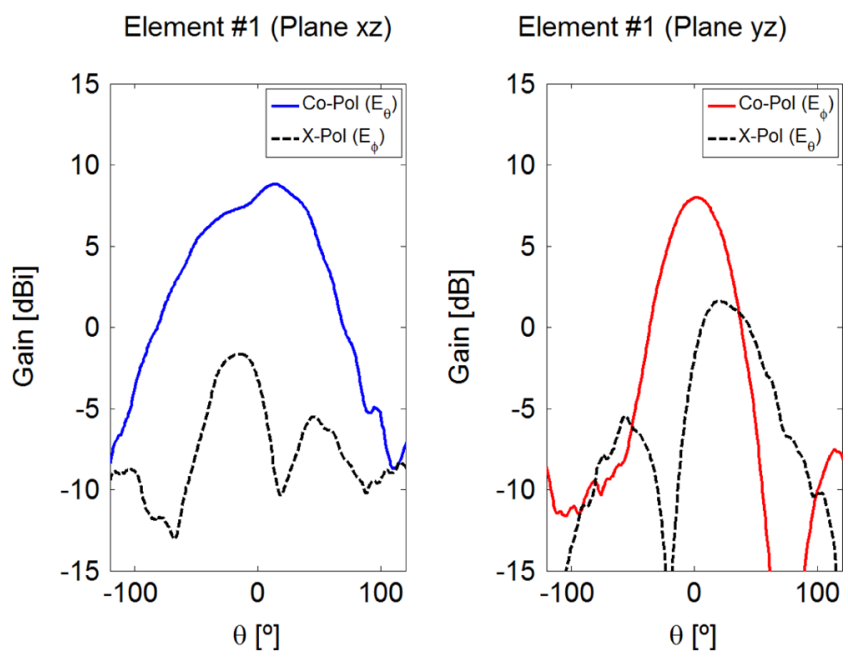

Fig. 6. Measured radiation pattern of the first element of the patches array at $2.6 \mathrm{GHz}$.

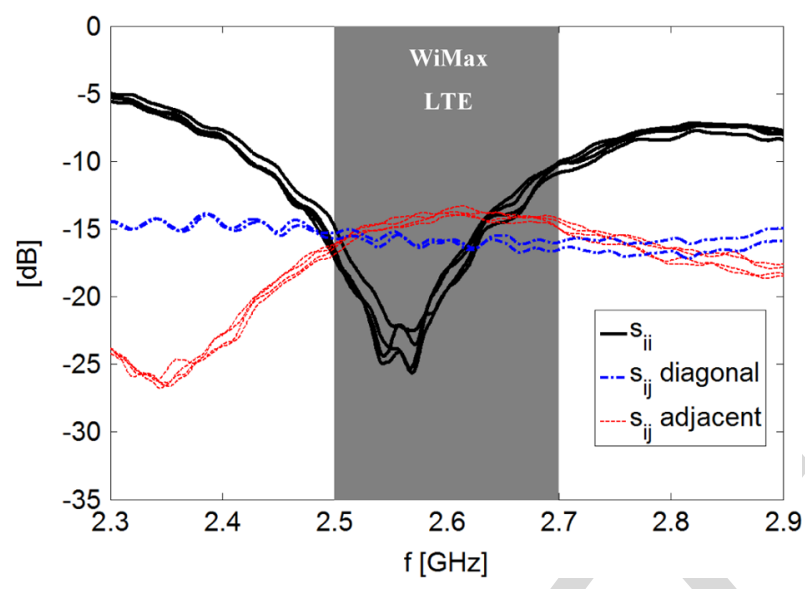

Fig. 7. Measured magnitude of the patch array scattering matrix elements.

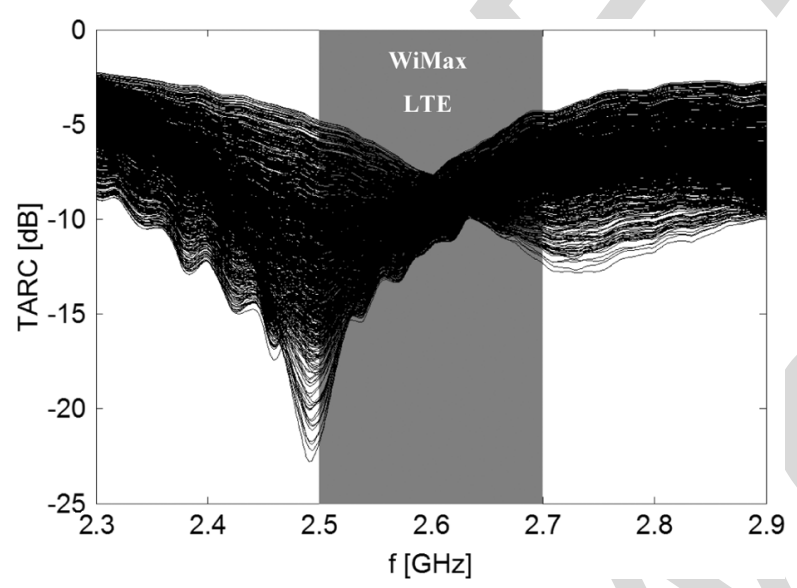

Fig. 8. Measured TARC for the patch array prototype.

\section{MIMO PERFORMANCE EVALUATION}

\section{A. Measurement Setup}

In order to fully test the non-ideal antenna effects in real MIMO environments, two identical arrays of each type were manufactured and tested using a channel sounder in a $4 \times 4$ link

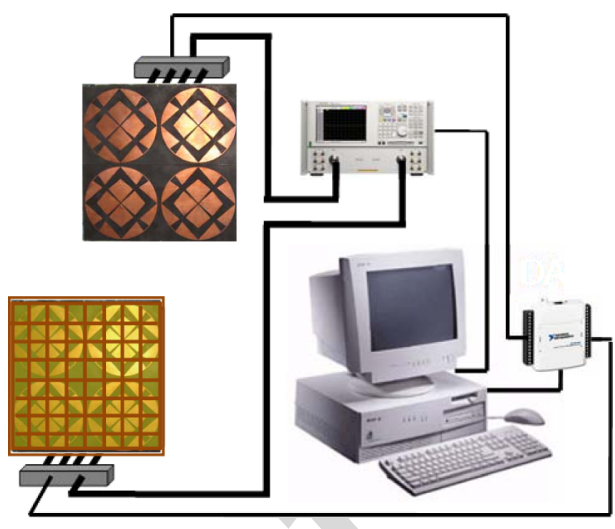

Fig. 9. MIMO measuring setup.

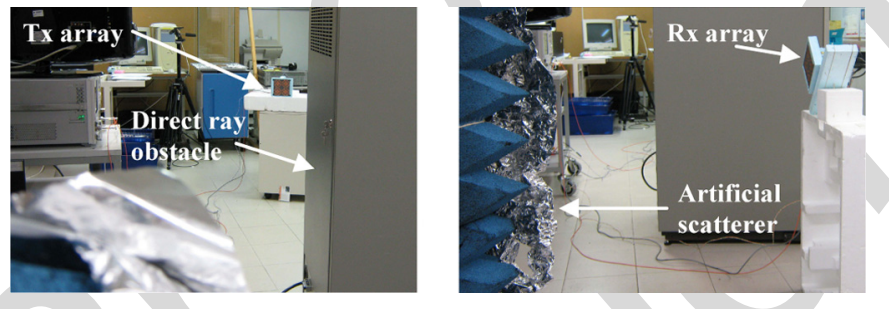

Fig. 10. MIMO measuring environment: (a) transmitting array; (b) receiving array.

configuration $\left(N_{\mathrm{t}}=4\right.$ and $\left.N_{r}=4\right)$, with equal Tx and Rx antennas. The setup is indicated in Fig. 9. A Vector Network Analyzer (Agilent E8361A) is used for measuring the MIMO channel transfer matrix $\mathbf{H}$ (with $N_{r} \times N_{t}$ elements). The two ports from the vector network analyzer (VNA) are electronically switched through all elements of the Tx and Rx antenna arrays. An in-house LabView application is used to remotely control the measurement set-up and data logging. The system enables to sequentially retrieve up to 16 channel responses (corresponding to every combination of $\mathrm{Tx}$ and $\mathrm{Rx}$ array elements, up to four elements in each array). Total acquisition time is of the order of 30 s.

An indoor laboratory was chosen as the measurement environment, Fig. 10. The Rx array was kept stationary at a fixed position, while the Tx array scanned a $1 \mathrm{~m} \times 1 \mathrm{~m}$ area with 10 $\mathrm{cm}$ mesh size, yielding $N_{p}=101$ measurement locations. Distance between Rx and Tx antennas was in the order of $4 \mathrm{~m}$. The channel frequency responses corresponding to each of the 16 $\mathrm{Rx} / \mathrm{Tx}$ array element combinations were measured in the 2.5 to $2.7 \mathrm{GHz}$ interval (WiMax and LTE bands) taking $N_{f}=201$ frequency samples.

To force non-line-of-sight link (NLOS) conditions, a metallic cabinet was used to block the direct ray path. It is well known that MIMO performance improvement is best evidenced for NLOS scenarios, where diversity gain is the highest, corresponding to most uncorrelated transmission channels [2]. In order to enhance multipath propagation, some artificial scatters were placed in the room, made from objects covered with ruffled aluminum foil.

Antenna coordinates and orientation, as well as cables, calibration procedures and scenario were exactly the same for both the CXETS array and the patch array. The environment was kept 

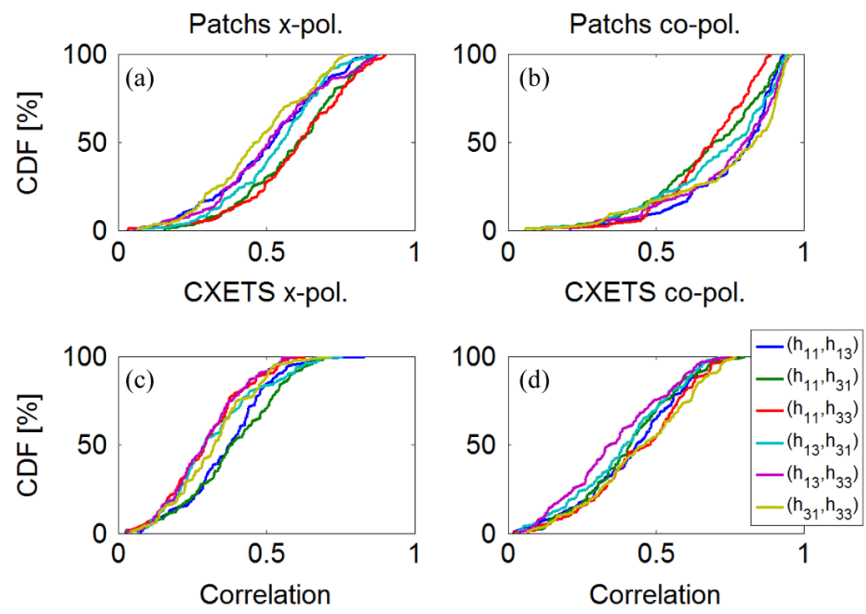

Fig. 11. Measured correlation between channels in a $2 \times 2$ MIMO setup using: (a) two cross-polarized patches (\#1 and \#2); (b) two co-polarized patches (\#1 and \#3); (c) two cross-polarized CXETS (\#1 and \#2); (d) two co-polarized CXETS (\#1 and \#3).

rigorously stationary throughout the whole measurement campaign, including the use of remote data logging to avoid operator influence on the scenario.

Measurements were performed with equal Tx and Rx antennas, instead of using a typical antenna at the personal terminal, because this allows isolating the contribution from the proposed CXETS antenna, without being limited or degraded by the characteristics of the personal terminal antenna.

\section{B. Capacity Estimation}

A first analysis of the measurements is performed using data from only two elements of the array, as in a $2 \times 2$ MIMO configuration $\left(N_{t}=2\right.$ and $\left.N_{r}=2\right)$. Either two cross-polarized array elements in both Tx and Rx are selected (data from antennas \#1 and \#2), or two co-polarized elements are selected (data from antennas \#1 and \#3). This analysis allows confirming the benefit from using polarization diversity and to evaluate how the CXETS and the patch arrays compare with respect to channel capacity in view of their different coupling characteristic.

Maximum MIMO performance requires parallel uncorrelated channels [16]. For the $2 \times 2$ configuration there are 4 parallel channels and therefore, 6 correlation coefficients $\rho$ can be determined between them

$$
\rho=\langle a, b\rangle=\left|\frac{E\left([a-E(a)][b-E(b)]^{*}\right)}{\sqrt{E\left(a^{2}\right)-E(a)^{2}} \sqrt{E\left(b^{2}\right)-E(b)^{2}}}\right|
$$

where $E(\cdot)$ corresponds to the expected value calculated over the measured bandwidth, $[\cdot] *$ represents the complex conjugate, and $a$ and $b$ represent measured channel responses between any two antenna pairs. All six correlation coefficients are determined for each of the $N_{p}=101$ measured positions inside the room. The corresponding cumulative distribution function (CDF) is represented in Fig. 11 for the CXETS array link and for the patch array link, considering either the crossor co-polarized antennas. Therefore, the plot vertical axis indicates the probability of finding a position in the room with channel correlation coefficient below the abscissa value.
The first observation is that for both polarization configurations, correlation is much lower for the CXETS, owing to its better inter-element isolation and polarization purity. Anyway, for both array types, the correlation coefficient is lower when cross-polarized antenna elements are used. This confirms that diversity gain increases when orthogonal polarized antennas are used [4]. It is noted that the decrease in channel correlation is achieved despite the co-polarized antennas being farther from each other as they are laid along the diagonal of the array and not adjacent to each other as with the cross-polarized ones, see Figs. 1 and 5.

Consider the case where only the receiver has channel information; the maximum achievable channel capacity $C$ is given by Shannon's equation [16]

$$
C=\log _{2}\left(I_{N_{r}}+\frac{S N R}{N_{t}} \mathbf{H}_{\mathbf{n}} \mathbf{H}_{\mathbf{n}}^{H}\right) \mathrm{bit} / \mathrm{s} / \mathrm{Hz}
$$

where $I$ is a $N_{r} \times N_{r}$ identity matrix, SNR is the signal-to-noise ratio at the receiver and $[.]^{H}$ represents the Hermitian operator. To exclude the effect of path loss variation on the received power, $\mathbf{H}_{\mathbf{n}}$ is normalized so that the average power is unitary [10], [17], [18]

$$
\mathbf{H}_{\mathbf{n}}=\frac{\mathbf{H}}{\sqrt{\frac{1}{N_{r} N_{t} N_{f} N_{p}} \sum_{N_{r}} \sum_{N_{t}} \sum_{N_{f}} \sum_{N_{p}}|\mathbf{H}|^{2}}} .
$$

Although this normalization removes path loss, it does not hide the effect of propagation-induced correlation or array element coupling-induced correlation. As previously mentioned, the non-normalized channel transfer matrix $\mathbf{H}$ corresponds directly to the $\mathbf{S}$-matrix measured at the Tx and Rx array ports, using a vector network analyzer [17].

Capacity values are calculated for the CXETS and patch arrays at $\mathrm{SNR}=10 \mathrm{~dB}$ for each frequency and position sample in different MIMO configurations, using (2) and the measured channel matrix. Fig. 12 presents the corresponding cumulative distribution function. For reference, Fig. 12 also shows for each MIMO configuration the calculated capacity for a Rayleigh fading channel, that is, each element $h_{n}$ of the normalized channel transfer matrix $\mathbf{H}_{\mathbf{n}}$ is given by

$$
h_{n}=\frac{1}{\sqrt{2}}[\operatorname{Normal}(0,1)+j \operatorname{Normal}(0,1)] .
$$

where $\operatorname{Normal}(0,1)$ is an independent and identically distributed (i.i.d.) value with Gaussian distribution function, zero mean and unit variance $\sigma_{h}^{2}=1$. This corresponds to totally uncorrelated channels.

Capacity improvement from the CXETS array against the array of patches is more evident for higher order MIMO configurations (Fig. 12(c)-(d)). This is clearer when comparing median capacity values (dashed horizontal line in Fig. 12). The results for each type of array are indicated in Table II along with the ratio between the capacity obtained from measured channel data 

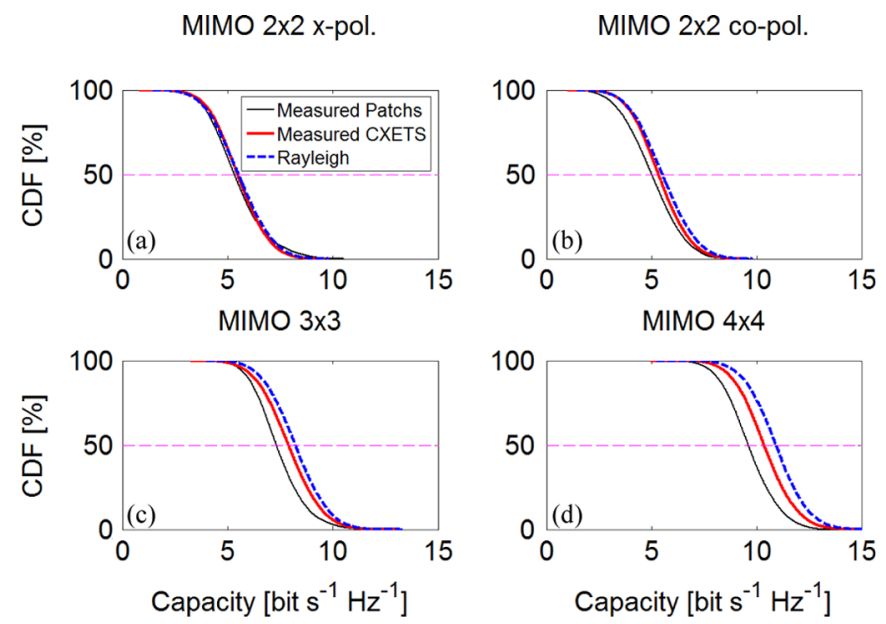

Fig. 12. Measured and simulated capacity for a MIMO set-up with $\mathrm{SNR}=10 \mathrm{~dB}$ at Rx and Tx, assuming power control : (a) two co-polarized array elements (b) two cross-polarized array elements; (c) three array elements; (d) all four array elements.

TABLE II

CAPACITY VALUES FOR 50\% PROBABILITY

\begin{tabular}{|c|c|c|c|c|c|}
\hline \multirow{2}{*}{$\#$} & & \multicolumn{2}{c|}{ Patchs } & \multicolumn{2}{c|}{ CXETS } \\
\cline { 3 - 6 } & & $\begin{array}{c}\text { Capacity } \\
{[\text { bit/s/Hz] }}\end{array}$ & $\begin{array}{c}\text { C } / \\
\mathrm{C}_{\text {Rayleigh }}\end{array}$ & $\begin{array}{c}\text { Capacity } \\
{[\text { bit/s/Hz }]}\end{array}$ & $\begin{array}{c}\mathrm{C} / \\
\mathrm{C}_{\text {Rayleigh }}\end{array}$ \\
\hline a & $2 \times 2 \times$ x-pol. & 5.33 & $96.65 \%$ & 5.48 & $99.46 \%$ \\
\hline b & $2 \times 2$ co-pol. & 5.01 & $90.93 \%$ & 5.34 & $96.96 \%$ \\
\hline c & $3 \times 3$ & 7.32 & $89.15 \%$ & 7.90 & $96.19 \%$ \\
\hline d & $4 \times 4$ & 9.62 & $87.78 \%$ & 10.36 & $94.60 \%$ \\
\hline
\end{tabular}

and the capacity calculated for completely independent channels (Rayleigh curves).

Table II shows that due to antenna mutual coupling and channel correlation, capacity does not scale linearly with number of channels as would be expected if they were independent [16]. In fact, the detrimental effect of mutual coupling on capacity increases with the number of array elements [15]. However it is clear in all cases the importance of better isolation and polarization purity of the CXETS.

These results assume that the system uses power control to fix SNR at constant $10 \mathrm{~dB}$ level. Otherwise, in environments with poor multipath richness, configuration \#b will tend to receive in average more power than configuration \#a. If we want to alternatively compare capacity for constant $T x$ power, we can consider for all cases of Table II the complete measured channel transfer matrix $\mathbf{H}\left(N_{r}=N_{t}=4\right)$ in the normalization (3) and extract afterwards the sub-matrixes from $\mathbf{H}_{\mathbf{n}}$ corresponding to each configuration [18]. For configuration \#a, this leads instead to $4.57 \mathrm{bit} / \mathrm{s} / \mathrm{Hz}$ for the patch array and $5.18 \mathrm{bit} / \mathrm{s} / \mathrm{Hz}$ for the CXETS array, which is not very different from the fixed SNR case thanks to the richness of the tested scenario. As could be expected, the other array configurations capacity remains practically unchanged.

For spatial multiplexed MIMO systems, it is relevant to further evaluate the eigenvalues of the measured $\mathbf{H}_{\mathbf{n}}$. Each eigenvalue represents an orthogonal parallel channel where independent data stream can be transmitted simultaneously, thus increasing the bit rate of the system [2]. Calculations were made
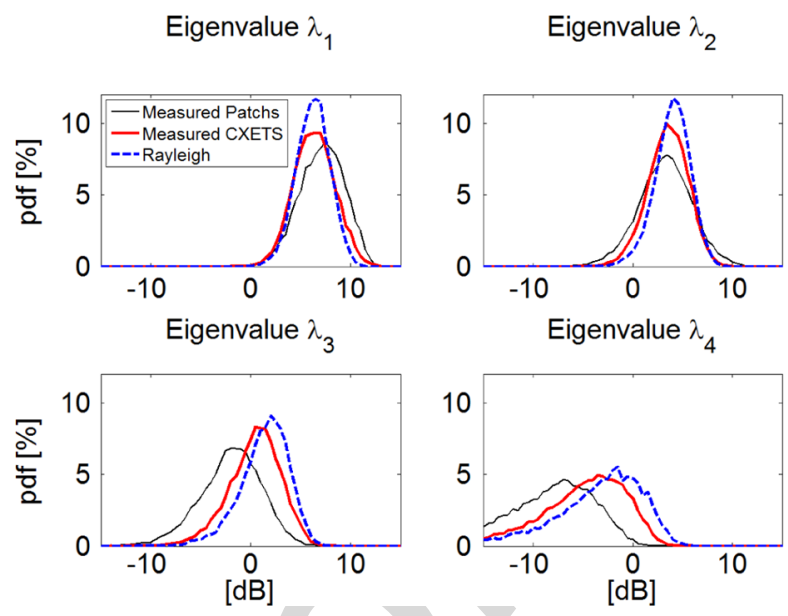

Fig. 13. Measured and simulated eigenvalues of the $4 \times 4$ MIMO setup.

for the $4 \times 4$ MIMO configuration $\left(N_{t}=4\right.$ and $\left.N_{r}=4\right)$, using all $\mathbf{H}_{\mathbf{n}}$ transfer functions measured at each frequency and at each array location in the room. For each measurement, the eigenvalues were ordered such that $\lambda_{1}>\lambda_{2}>\lambda_{3}>\lambda_{4}$. Fig. 13 presents the corresponding probability density functions considering $0.5 \mathrm{~dB}$ eigenvalue amplitude steps (bins).

As the level of correlation increases from the ideal Rayleigh channel case to the measured CXETS and patch array cases, the mean value of the first eigenvalue $\lambda_{1}$ increases while the mean of the other eigenvalues decreases. Such effect reflects the degradation of spatial multiplexed MIMO performance and the tendency to approximate the case of a single independent information channel, as in a SISO configuration. Again the CXETS array performance is much closer to the uncorrelated channel case (Rayleigh curves) than the array of patches due to much lower mutual coupling.

Shannon's (2) determines the maximum achievable capacity, but the actual throughput in a real system depends on further aspects like modulation and coding. A system emulator developed in Matlab textregistered was used to evaluate the simultaneous transmission of four independent and parallel bit streams using the measured channel transfer matrix in $4 \times 4$ MIMO configuration $\left(N_{\mathrm{t}}=4\right.$ and $\left.N_{r}=4\right)$. For instance using binary phase shift keying (BPSK) modulation and maximum-likelihood (ML) detection at the receiver, the transmission between two CXETS arrays presents a bit error rate (BER) at SNR $=10 \mathrm{~dB}$ that is almost 15 times lower than the equivalent configuration using two arrays of patches.

\section{CONCLUSION}

The proposed CXETS antenna array, with its very low coupling between the closely packed array elements and with its pure linear polarization characteristics, is shown to clearly enhance the known benefits from using space and polarization diversity in MIMO systems, when compared to commonly used antennas, like the array of patches. The large operating bandwidth from 2.4 to $4.8 \mathrm{GHz}$ with stable radiation characteristics allows using the CXETS array for multisystem access points covering services like WiFi, WiMax, LTE and UWB. The antenna cavity back increases the front to back radiation level 
above $12 \mathrm{~dB}$ allowing its mounting on metal surfaces or against a wall for WLAN access points. These cumulative characteristics make CXETS a unique and effective candidate for a very wideband MIMO array.

In order to test its advantage for MIMO applications, basic MIMO performance comparison was conducted at $2.6 \mathrm{GHz}$ (WiMax or LTE bands) between the CXETS array and a narrowband patch array with the same spatial and polarization configuration. Based upon measured channel transfer matrix using a MIMO channel sounder, it was shown that the new array increases channel capacity, better approaching the ideal limit for completely uncorrelated channels.

Although all MIMO performance tests were conducted only at the lower fraction of the CXETS available bandwidth, it is expected that the overall performance will improve further for the rest of the band. In fact, while the antenna radiation characteristics remain almost unchanged across the whole band, for higher frequencies the separation between CXETS array elements becomes larger in terms of the operating wavelength and, therefore, the channels between transmit and receive antennas are expected to become more uncorrelated.

\section{ACKNOWLEDGMENT}

The authors acknowledge the collaboration from V. Fred and C. Brito for prototype construction, and A. Almeida for prototype measurements.

\section{REFERENCES}

[1] S. M. Alamouti, "A simple transmit diversity technique for wireless communications," IEEE J. Sel. Areas Commun., vol. 16, pp. 1451-145\&I Oct. 1998.

[2] D. Tse and P. Viswanath, Fundamentals of Wireless Communication. Cambridge, U.K.: Cambridge Univ. Press, 2005.

[3] R. Janaswamy, "Effect of element mutual coupling on the capacity of fixed length linear arrays," IEEE Antennas Wireless Propag. Lett., vol. 1, pp. 157-160, 2002.

[4] J. Valenzuela-Valdés, M. García-Fernández, A. Martínez-González, and D. Sánchez-Hernández, "The role of polarization diversity for MIMO systems under Rayleigh-fading environments," IEEE Antennas Wireless Propag. Lett., vol. 5, pp. 534-536, 2006.

[5] J. Costa, C. Medeiros, and C. Fernandes, "Performance of a crossed exponentially tapered slot antenna for UWB systems," IEEE Trans. Antennas Propag., vol. 57, pp. 1345-1352, May 2009.

[6] C. Medeiros, J. Costa, and C. Fernandes, "Compact tapered slot UWB antenna with WLAN band rejection," IEEE Antennas Wireless Propag. Lett., vol. 8, pp. 661-664, 2009.

[7] C. Medeiros, E. Lima, J. Costa, and C. Fernandes, "Wideband slot antenna for WLAN access points," IEEE Antennas Wireless Propag. Lett., vol. 9, pp. 79-82, 2010.

[8] R. Bhatti, J. Choi, and S. Park, "Quad-band MIMO antenna array for portable wireless communications terminals," IEEE Antennas Wireless Propag. Lett., vol. 8, pp. 129-132, 2009.

[9] H. Li, J. Xiong, and S. He, "Extremely compact dual-band PIFAs for MIMO application," Electronics Lett., vol. 45, pp. 869-870, Aug. 2009.

[10] A. Rajagopalan, G. Gupta, A. Konanur, B. Hughes, and G. Lazzi, "Increasing channel capacity of an ultrawideband MIMO system using vector antennas," IEEE Trans. Antennas Propag., vol. 55, pp. 2880-2887, Oct. 2007.

[11] S. Zhang, Z. Ying, J. Xiong, and S. He, "Ultrawideband MIMO/diversity antennas with a tree-like structure to enhance wideband isolation," IEEE Antennas Wireless Propag. Lett., vol. 8, pp. 1279-1282, 2009.
[12] S. Zhang, P. Zetterberg, and S. He, "Printed MIMO antenna system of four closely-spaced elements with large bandwidth and high isolation," Electronics Lett., vol. 46, pp. 1052-1053, July 2010.

[13] M. Manteghi and Y. Rahmat-Samii, "Multiport characteristics of a wide-band cavity backed annular patch antenna for multipolarization operations," IEEE Trans. Antennas Propag., vol. 53, pp. 466-474, Jan. 2005.

[14] [Online]. Available: www.cst.comCST [Online]. Available:

[15] D. Browne, M. Manteghi, M. Fitz, and Y. Rahmat-Samii, "Experiments with compact antenna arrays for MIMO radio communications," IEEE Trans. Antennas Propag., vol. 54, pp. 3239-3250, Nov. 2006.

[16] G. Foschini and M. Gans, "On limits of wireless communications in a fading environment when using multiple antennas," Wireless Personal Commun., vol. 6, pp. 311-335, 1998.

[17] M. Herdin, H. Ozcelik, H. Hofstetter, and E. Bonek, "Variation of measured indoor MIMO capacity with receive direction and position at 5.2 GHz," Electron. Lett., vol. 38, pp. 1283-1285, Oct. 2002.

[18] J. Wallace, M. Jensen, A. Swindlehurst, and B. Jeffs, "Experimental characterization of the MIMO wireless channel: Data acquisition and analysis," IEEE Trans. Wireless Commun., vol. 2, pp. 335-343, Mar. 2003.

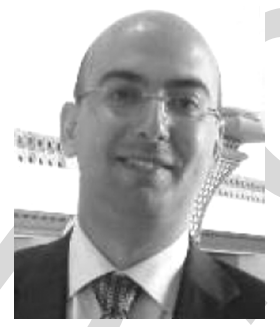

Jorge R. Costa (S'97-M'03-SM'09) was born in Lisbon, Portugal, in 1974. He received the Licenciado and Ph.D. degrees in electrical and computer engineering from the Instituto Superior Técnico (IST), Technical University of Lisbon, Lisbon, Portugal, in 1997 and 2002, respectively.

$\mathrm{He}$ is currently a Researcher at the Instituto de Telecomunicações, Lisbon, Portugal. He is also an Assistant Professor at the Departamento de Ciências e Tecnologias da Informação, Instituto Superior de Ciências do Trabalho e da Empresa. His present research interests include lenses, reconfigurable antennas, MEMS switches, UWB, MIMO and RFID antennas. He is the coauthor of four patent applications and more than 50 contributions to peer reviewed journals and international conference proceedings. More than ten of these papers have appeared in IEEE Journals.

Prof. Costa is currently serving as an Associate Editor for the IEEE TRANSACTIONS ON ANTENNAS AND PROPAGATION.

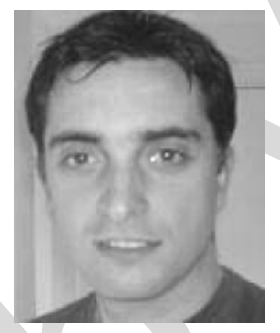

Eduardo B. Lima was born in Viseu, Portugal, in 1978. He received the Licenciado and M.Sc. degrees in electrical and computer engineering from the Instituto Superior Técnico (IST), Lisbon, Portugal, in 2003 and 2008, respectively.

$\mathrm{He}$ is a Researcher and also the software developer for antenna measurements control at the Instituto de Telecomunicações, Lisbon, Portugal. From 2004 to 2007, he was involved on the ESA/ESTEC project ILASH (Integrated Lens Antenna Shaping). He is the coauthor of one patent application and twelve technical papers in international journals and conference proceedings in the area of antennas. His present research interests include dielectric lens antennas and MIMO.

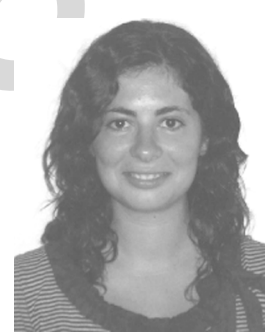

Carla R. Medeiros was born in Ponta Delgada, Açores, Portugal, in 1982. She received the Licenciado and M.Sc. degrees in electrical and computer engineering from the Instituto Superior Técnico (IST), Technical University of Lisbon, Lisbon, Portugal, in 2006 and 2007, respectively.

Since 2006, she has been a Researcher at the Instituto de Telecomunicações (IT), focusing her work on antenna for wireless communications. She collaborates in national research projects. Her current research interests are in the areas of reconfigurable, RFID, MIMO and UWB antennas. 


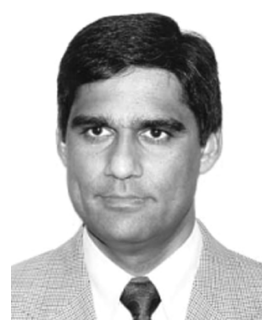

Carlos A. Fernandes (S'86-M'89-SM'08) received the Licenciado, M.Sc., and Ph.D. degrees in electrical and computer engineering from Instituto Superior Técnico (IST), Technical University of Lisbon, Lisbon, Portugal, in 1980, 1985, and 1990, respectively.

He joined the IST in 1980, where he is presently Full Professor at the Department of Electrical and Computer Engineering in the areas of microwaves, radio wave propagation and antennas. He is a Senior Researcher at the Instituto de Telecomunicações and member of the Board of Directors. He has been the Leader of antenna activities in National and European Projects as RACE 2067-MBS (Mobile Broadband System), ACTS AC230-SAMBA (System for Advanced Mobile Broadband Applications) and ESA/ESTEC-ILASH (Integrated Lens Antenna Shaping). He has coauthored a book, a book chapter, and more than 120 technical papers in peer reviewed international journals and conference proceedings, in the areas of antennas and radiowave propagation modeling. His current research interests include dielectric antennas for millimeter wave applications, antennas and propagation modeling for personal communication systems, RFID antennas, artificial dielectrics and metamaterials. 


\title{
Evaluation of a New Wideband Slot Array for MIMO Performance Enhancement in Indoor WLANs
}

\author{
Jorge R. Costa, Senior Member, IEEE, Eduardo B. Lima, Carla R. Medeiros, and \\ Carlos A. Fernandes, Senior Member, IEEE
}

\begin{abstract}
A new wideband compact slot antenna array for indoor WLAN access points (AP) is described, covering several wireless communication services from 2.4 to $4.8 \mathrm{GHz}$, that is especially designed to enhance MIMO system capacity. The array topology provides both spatial and polarization diversity. Despite very close packing of the array elements, these exhibit very low mutual coupling and low cross-polarization, greatly favoring MIMO diversity gain. A detailed MIMO performance comparison is conducted against a common array of patches in indoor environment, based both on simulation and indoor measurements: the new antenna shows a clear improvement in terms of channel capacity.
\end{abstract}

Index Terms-Printed wideband antenna, tapered slot antenna, multiple input multiple output (MIMO) array, wireless local area network (WLAN), spatial and polarization diversity.

\section{INTRODUCTION}

$\mathbf{T}$ HE benefits from using multiple input and multiple output antennas (MIMO) in wireless communications have been widely discussed in the literature. Spatial diversity improves system reliability by decreasing the sensitivity to fading (diversity gain) without requiring additional bandwidth, unlike the case of frequency or time diversity. Spatial multiplexing can be implemented as an alternative to spatial diversity. In spatial multiplexing each $N_{t}$ transmitter antenna sends an independent data stream, unlike the case of spatial diversity where each antenna sends correlated data (e.g., Alamouti code [1]). With spatial multiplexing the spectral efficiency can be increased by a factor $\min \left(N_{t}, N_{r}\right)$ [2].

Both for spatial diversity and for spatial multiplexing strategies, it is required that the channels between the multiple transmitting $(\mathrm{Tx})$ and receiving $(\mathrm{Rx})$ antennas are uncorrelated to maximize data throughput and for successful data stream decoding. Correlation may result from poor multipath richness of the scenario and from mutual coupling between antenna array elements [3]. Therefore, one important design specification for MIMO antenna arrays is to minimize inter-element coupling.

Manuscript received March 19, 2010; revised August 20, 2010; accepted September 02, 2010. Date of publication January 31, 2011; date of current version April 06, 2011. This work was supported in part by the European Union under project FP7 ICT-2007-216715 (NewCom++).

J. R. Costa is with the Instituto de Telecomunicações, IST, 1049-001 Lisboa, Portugal and also with the Departamento de Ciências e Tecnologias da Informação, ISCTE-Lisbon University Institute, 1649-026 Lisboa, Portugal (e-mail: Jorge.Costa@1x.it.pt).

E. B. Lima, C. R. Medeiros, and C. A. Fernandes are with Instituto de Telecomunicações, IST, 1049-001 Lisboa, Portugal .

Color versions of one or more of the figures in this paper are available online at http://ieeexplore.ieee.org.

Digital Object Identifier 10.1109/TAP.2011.2109685
Polarization diversity was found to be as significant as spatial diversity at improving diversity gain, and hence MIMO system capacity [4]. Therefore, MIMO array elements should also present pure and frequency stable polarization to obtain full benefit from polarization diversity.

The present study aims to present and evaluate a $2 \times 2$ printed array antenna intended to enhance MIMO performance in indoor access points (fixed terminal), with the following cumulative characteristics: low inter-element coupling, pure linear polarization from each array element, good front-to-back ratio for wall mounting, high radiation efficiency, and stability of these characteristics over a large bandwidth to accommodate multiple wireless standards such as WiFi, LTE, WiMAX and part of the UWB band. Furthermore, the four array elements are to be arranged in a compact layout with space and polarization diversity.

The proposed array elements are based upon the linear polarized crossed exponentially tapered slot (XETS) antenna developed by the authors for ultrawideband (UWB) systems [5], [6] and modified in [7] to include a back cavity to change the inherently bidirectional radiation pattern into a unidirectional one. This new configuration is referred as cavity back crossed exponentially tapered slot (CXETS) antenna and presents 67\% bandwidth. The antenna copes with all the specifications listed above and confirmed MIMO improved performance when compared by simulation and measurement to a reference printed patch array with the same element layout and diversity polarization scheme.

To the authors' knowledge, no other antenna reported in the literature copes simultaneously with all the above specifications, which makes the CXETS array a novel alternative for MIMO fixed terminal. Many MIMO arrays were proposed in the literature, but in all cases they match only part of the above requirements [8]-[13], and clearly no solution cumulatively presents comparable characteristics.

This paper is organized as follows. The new CXETS array and the array of patches that is used for comparison are both presented in Section II. Section III describes both the MIMO setup for channel transfer matrix measurements and the performance evaluation of several MIMO configurations with different number of array elements based upon channel capacity estimation. Numerical and experimental results are given and discussed throughout. Conclusions are drawn in Section IV.

\section{ARRAY DESCRIPTION}

Both the XETS and rectangular patch arrays were designed using the transient solver of CST Microwave Studio ${ }^{\mathrm{TM}}$, based on Finite Integration Method [14]. To ensure fair performance 


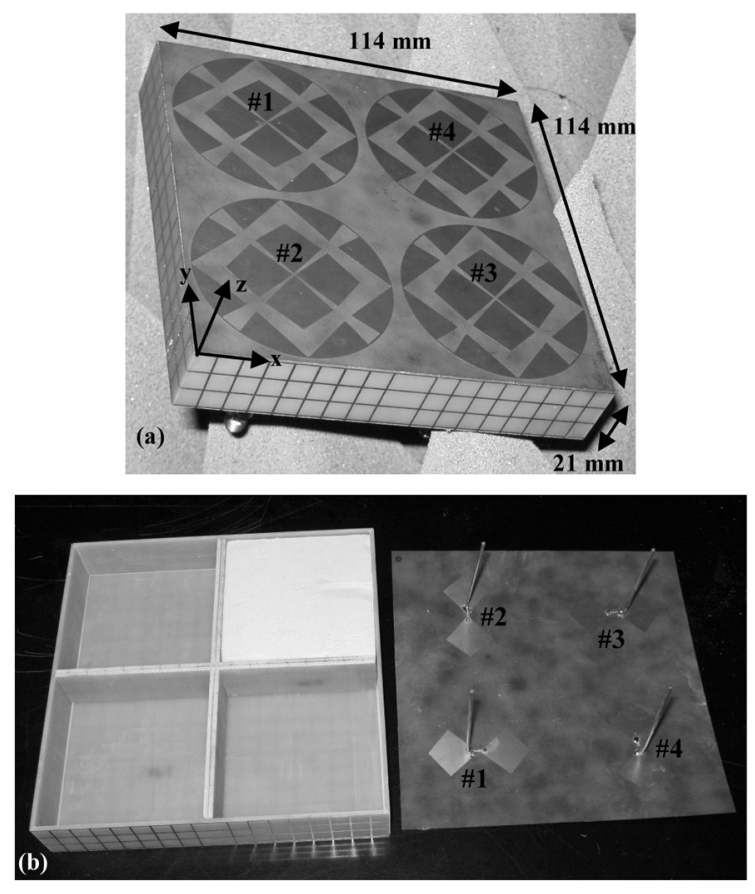

Fig. 1. (a) Photograph of the assembled CXETS array prototype, showing the antenna top face; (b) Disassembled antenna showing the FR4 cavity back and the feeding coaxial cables soldered on the XETS back petals.

comparison between both arrays, they were designed to have similar aperture area.

\section{A. CXETS Array}

The crossed exponentially tapered slot (XETS) antenna configuration was developed by the authors for UWB systems [5], [6]. It is compact, with stable radiation pattern, low cross-polarization and low mutual coupling when packed in a closed array configuration. However, the radiation pattern is bidirectional. To produce a unidirectional radiation pattern, a single antenna was redesigned to include a grid back cavity [7]. This new configuration is onwards referred as the CXETS.

The XETS elements are printed on both sides of DUROID 5880 substrate, $\varepsilon_{r}=2.2, \tan (\delta)=0.0009$ and thickness $h_{5880}=10 \mathrm{mil}=0.254 \mathrm{~mm}$. The top face of the four radiating elements is shown in Fig. 1(a) and the back face, with the feeding coaxial cables, is shown in Fig. 1(b). These are small diameter EZ-47 semi-rigid coaxial cables (1.19 mm diameter), soldered between two replicas of opposing petals from the front face of each XETS, Fig. 1(b). The RF signal is capacitively coupled to the corresponding front petals of each antenna. The fed petals define the E-plane of each XETS. It was verified that common-mode currents resulting from the unbalanced feeding are low in this assembly and do not perturb significantly the expected balanced antenna performance. It is seen in Fig. 1(b) that adjacent elements have orthogonal orientation, so, orthogonal polarization.

The meshed cavity walls are printed on FR4 substrate, $\varepsilon_{r}=$ $4.9, \tan (\delta)=0.025$ and thickness $h_{\mathrm{FR} 4}=1.6 \mathrm{~mm}$. The mesh is uniform, with $6 \mathrm{~mm}$ step. This is a compromise value found through CST simulation that allows retaining a reasonable part of the original antenna bandwidth (now 2.4 to $4.8 \mathrm{GHz}$ ) and at
TABLE I

CXETS ANTENNA PARAMETER VALUES IN MiLlimeters

\begin{tabular}{|c|c|c|c|c|c|c|}
\hline$D_{s}$ & $L_{\text {out }}$ & $L_{\text {in }}$ & $w_{s}$ & $L$ & $w_{0}$ & $C_{0}$ \\
\hline 54 & 35.7 & 27.9 & 9 & 53 & 0.72 & 12.7 \\
\hline
\end{tabular}

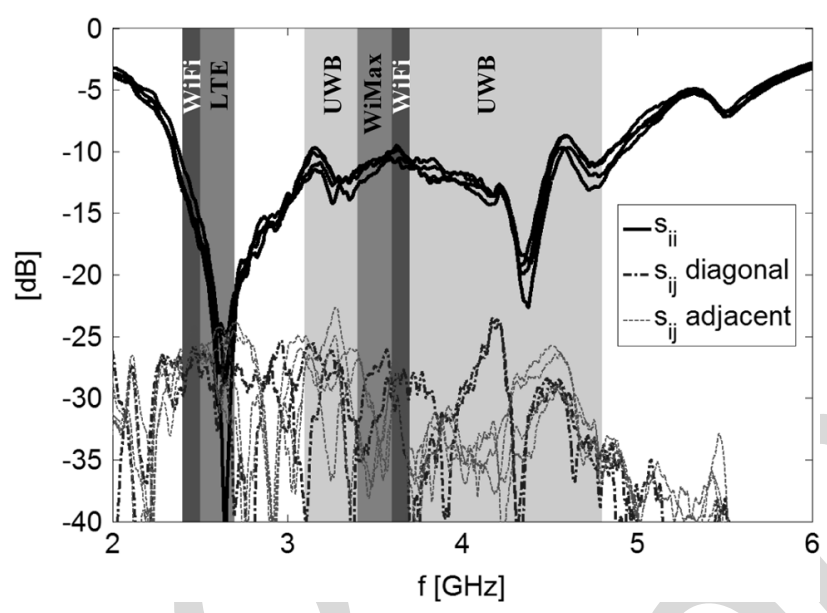

Fig. 2. Measured amplitude of the CXETS array scattering matrix elements.

the same time enables reaching better than $10 \mathrm{~dB}$ front-to-back ratio (f/b). Each array element has its own cavity; the two walls separating each cavity can be observed in Fig. 1(b). The metallic mesh is also printed on the metallic walls. In the assembled antenna, the cavities are filled with low density Styrofoam $\left(\varepsilon_{r} \approx\right.$ $1.05)$ to provide physical support to the thin 10 mils DUROID substrate. The Styrofoam can be observed in one of the cavities of the disassembled antenna in Fig. 1(b).

The remaining parameter values are indicated Table I (the same parameter naming is used as in [5]). The overall size of the array (Fig. 1(a)) is $114 \times 114 \times 21 \mathrm{~mm}^{3}\left(0.91 \lambda_{\text {inf }} \times 0.91 \lambda_{\text {inf }} \times\right.$ $0.17 \lambda_{\text {inf }}$ ) where $\lambda_{\text {inf }}$ is the wavelength at $2.4 \mathrm{GHz}$, the lower operating frequency. Smaller MIMO antenna assemblies have been reported for handheld communication devices, but none of those antennas offer 1:2 bandwidth. The proposed antenna is intended specifically for WLAN access points (fixed terminal).

Fig. 2 presents the measured scattering matrix of the CXETS array. The input reflection coefficient of all elements is below $-10 \mathrm{~dB}$ across the interval from 2.4 to $4.8 \mathrm{GHz}$. This covers WiFi, LTE, WiMax and the lower channels of UWB systems. The corresponding assigned bands are indicated in Fig. 2. It is seen from Fig. 2 that mutual coupling between array elements is mostly below $-25 \mathrm{~dB}$ across the entire antenna bandwidth. It is noted that the original XETS antenna presents very low coupling (about $-70 \mathrm{~dB}$ ) between adjacent cross-polarized array elements [5]. It is the addition of the back cavity that increases mutual coupling to $-25 \mathrm{~dB}$, but this value is still attractive for the present application.

It is known that a N-port antenna efficiency is better characterized by the total active reflection coefficient (TARC) [13], [15] than by the usual inspection of the $\mathbf{S}$-matrix elements. TARC is the square root of the ratio between total reflected power at the array ports and total power fed to the ports. It is calculated by applying a unity magnitude voltage with random phase at each port of the array. In this way, the reflected power translates different combinations of the excitation signals, which is more 


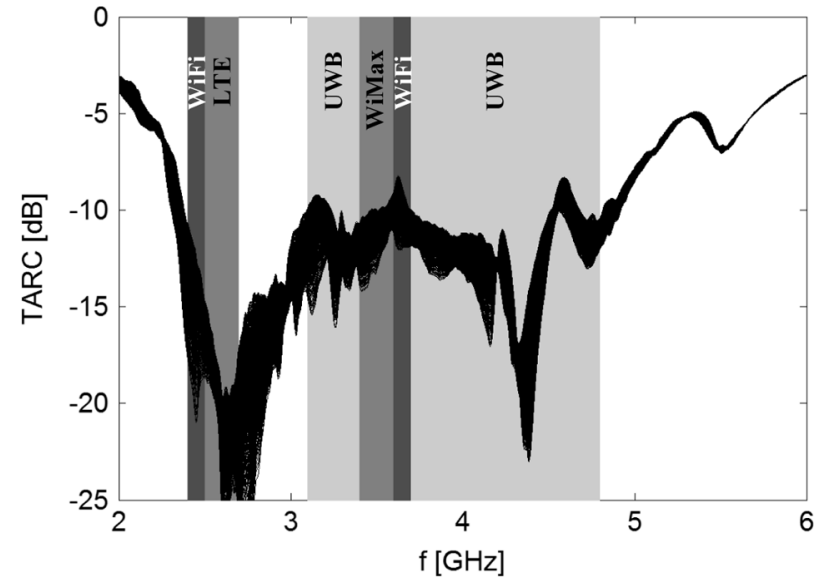

Fig. 3. Measured TARC for the CXETS array prototype.
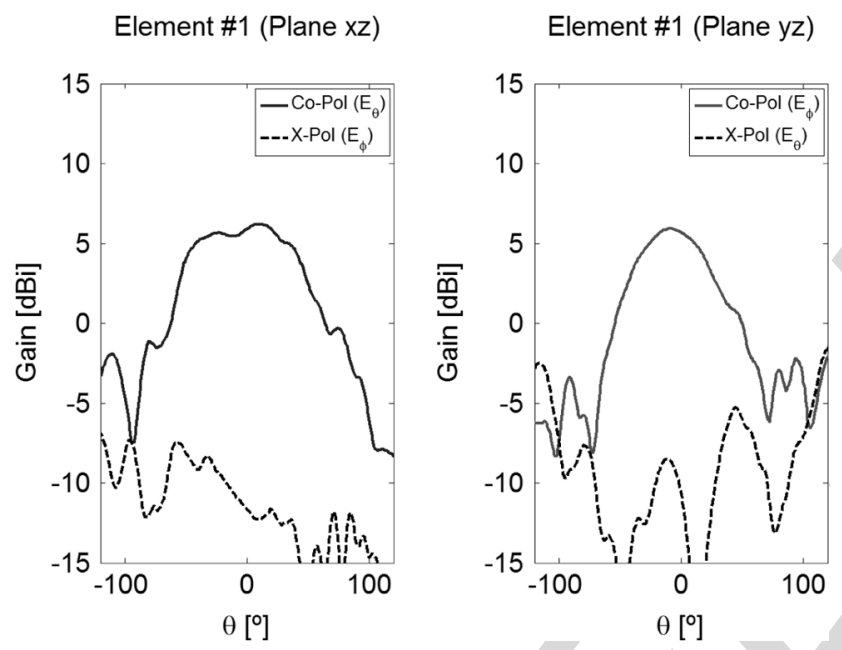

Fig. 4. Measured radiation pattern of the first element of the CXETS array at $2.6 \mathrm{GHz}$.

appropriate to describe the real conditions of a MIMO array operation than the usual single port excitation strategy. It accounts for both coupling and random signal combining between each port signal [13].

The measured scattering matrix of the CXETS was used to compute the antenna TARC. The results for different combinations of the input signals are presented in Fig. 3. Since the CXETS elements present low mutual coupling, TARC level is almost always below $-10 \mathrm{~dB}$ across the bandwidth.

The measured main planes radiation pattern of array element \#1 is presented in Fig. 4, with the other CXETS terminated by matched loads. Vertical scale refers to antenna gain. Cross-polarization level is about $-15 \mathrm{~dB}$. The other three array elements present similar radiation patterns, apart from plane interchange for the $90^{\circ}$ rotated antennas.

The measured front-to-back ratio across the bandwidth varies between 11 and $20 \mathrm{~dB}$ while gain ranges from 5.5 to $8 \mathrm{dBi}$. Cross-polarization improves with frequency from $-15 \mathrm{~dB}$ at 2.4 $\mathrm{GHz}$ to about $-20 \mathrm{~dB}$ at $4.8 \mathrm{GHz}$. CST simulations indicate that the array radiation efficiency is always above $80 \%$.
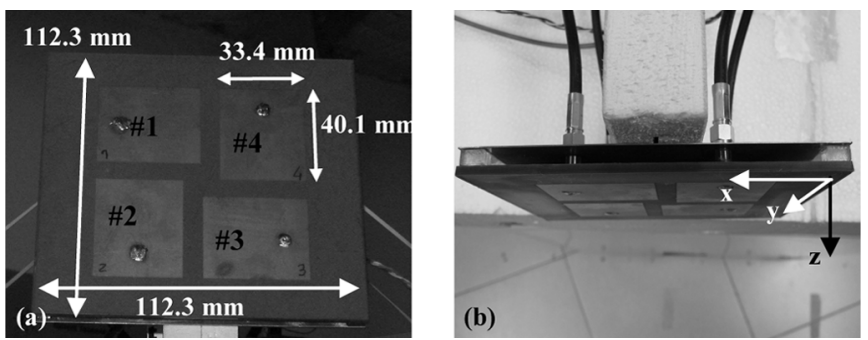

Fig. 5. (a) Photograph of the rectangular patch array prototype; (b) photograph of the air subtract and coaxial feeding.

\section{B. Rectangular Patch Array}

The rectangular patch array prototype is printed on DUROID $5880, \varepsilon_{r}=2.2, \tan (\delta)=0.0009$, thickness $h_{5880}=125 \mathrm{mil}=$ $3.175 \mathrm{~mm}$, see Fig. 5. It was designed to operate in the WiMax or LTE band from 2.5 to $2.7 \mathrm{GHz}$. The desired bandwidth was achieved through the use of an air gap between the dielectric layer and the ground plane $h_{\text {air }}=5 \mathrm{~mm}$. Of course it is not possible to approach the wide CXETS bandwidth with a conventional patch antenna. Patch dimensions $W_{P} \times L_{P}$ are 33.4 $\mathrm{mm} \times 40.1 \mathrm{~mm}\left(0.27 \lambda_{\text {inf }} \times 0.32 \lambda_{\text {inf }}\right)$. Each patch is probe fed by an EZ-141 coaxial cable (3.58 mm diameter). The outer conductor of the feeding coaxial cable is soldered to the ground plane and extended up to the dielectric; this arrangement reduces the excitation of surface waves that can propagate along the air gap and therefore lowers mutual coupling between elements as will be seen next. Patches are rotated by $90^{\circ}$ degrees in relation to the adjacent one, Fig. 5. Therefore, the polarization from the patches in one diagonal of the array is orthogonal with respect to the polarization from the other two patches. The ground plane extends by $15.9 \mathrm{~mm}$ from the edges of the patches. With $7.3 \mathrm{~mm}$ gap between adjacent elements, the overall array size is $112.3 \mathrm{~mm} \times 112.3 \mathrm{~mm}\left(0.9 \lambda_{\text {inf }} \times 0.9 \lambda_{\text {inf }}\right)$.

Fig. 6 presents the measured radiation pattern at $2.6 \mathrm{GHz}$ from array patch \#1, with the other patches terminated by matched loads. Vertical scale refers to antenna gain. Cross polarization is high as expected. Very similar radiation patterns were measured for the other three array elements, apart from plane interchange for orthogonal patches.

Fig. 7 presents the measured magnitude of the array scattering matrix elements. Grey shading represents the WiMax or LTE bands. Coupling between adjacent patches reaches $-13.5 \mathrm{~dB}$ within the WiMax or LTE band while coupling between diagonal patches is almost constant across the band at $-15 \mathrm{~dB}$ level. This is an improvement with respect to the usual configuration where the outer conductor of the feeding coaxial cable is terminated at the ground plane. In fact, CST simulations indicate mutual coupling level of $-10 \mathrm{~dB}$ in this case.

Fig. 8 presents TARC curves computed from the measured S-matrix, for random phase and unit amplitude at the excitation ports. It can be observed that the worst value within the WiMax or LTE bands is about $-5 \mathrm{~dB}$. The large dispersion of the TARC curves denotes a strong coupling between antenna elements, unlike the CXETS. 

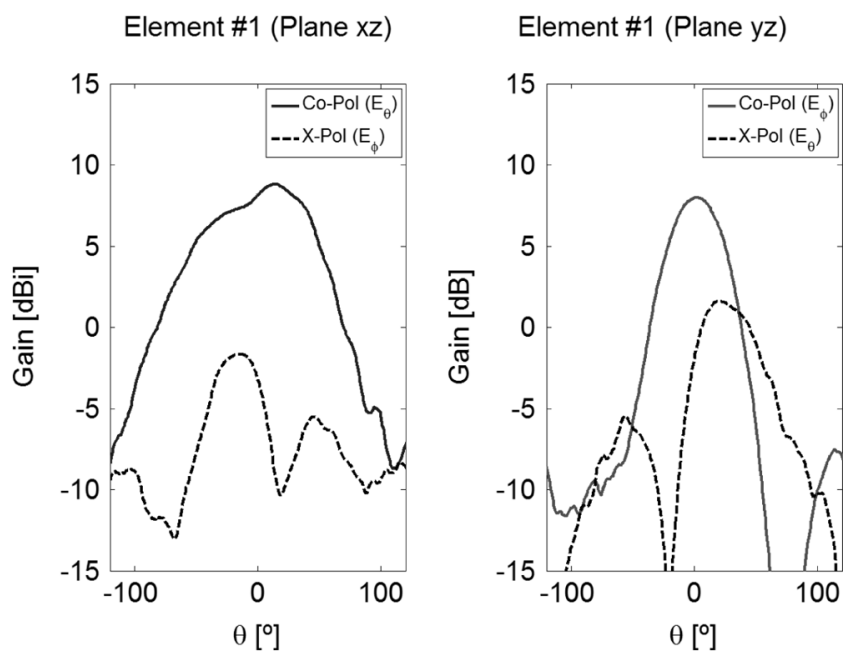

Fig. 6. Measured radiation pattern of the first element of the patches array at $2.6 \mathrm{GHz}$.

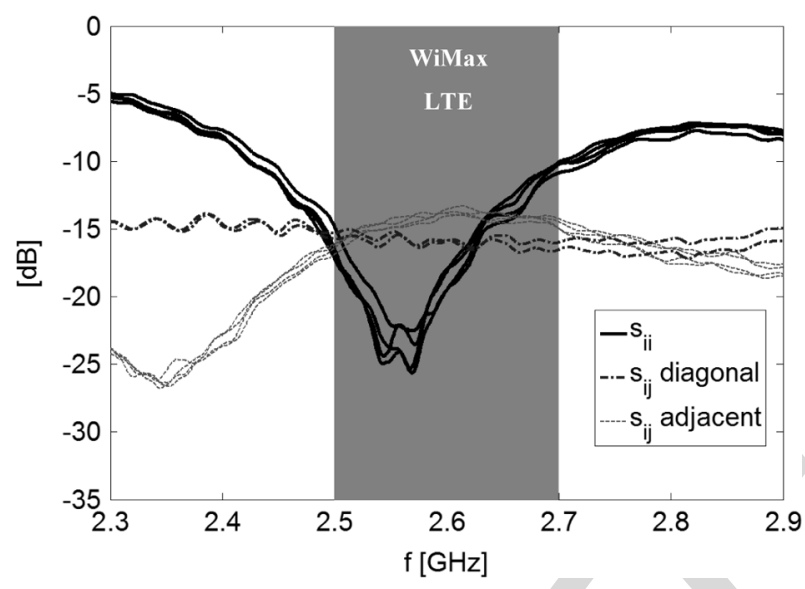

Fig. 7. Measured magnitude of the patch array scattering matrix elements.

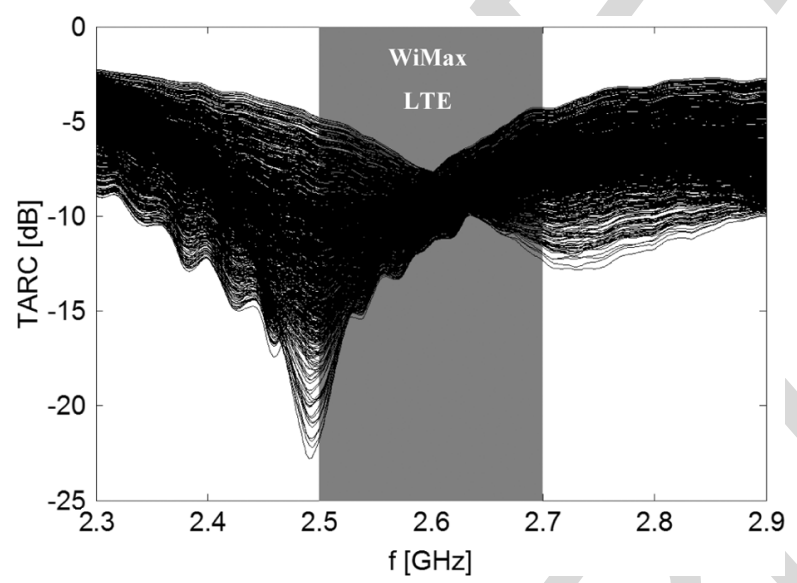

Fig. 8. Measured TARC for the patch array prototype.

\section{MIMO PERFORMANCE EVALUATION}

\section{A. Measurement Setup}

In order to fully test the non-ideal antenna effects in real MIMO environments, two identical arrays of each type were manufactured and tested using a channel sounder in a $4 \times 4$ link

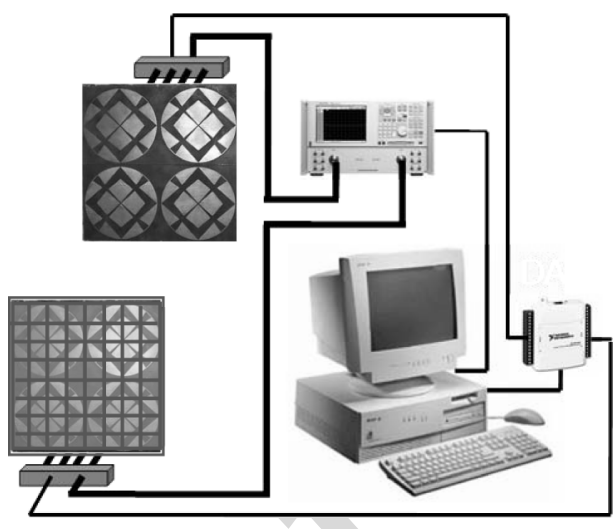

Fig. 9. MIMO measuring setup.
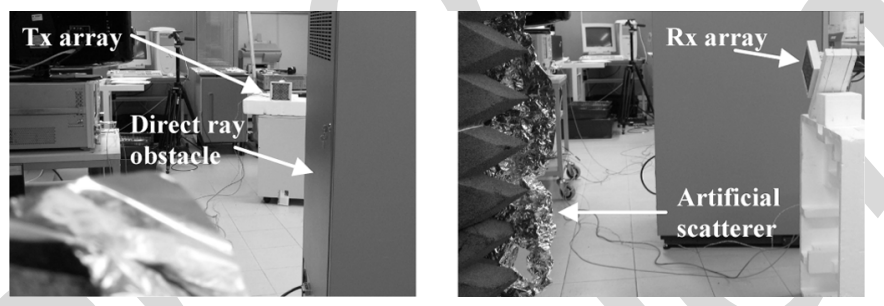

Fig. 10. MIMO measuring environment: (a) transmitting array; (b) receiving array.

configuration ( $N_{\mathrm{t}}=4$ and $N_{r}=4$ ), with equal Tx and Rx antennas. The setup is indicated in Fig. 9. A Vector Network Analyzer (Agilent E8361A) is used for measuring the MIMO channel transfer matrix $\mathbf{H}$ (with $N_{r} \times N_{t}$ elements). The two ports from the vector network analyzer (VNA) are electronically switched through all elements of the Tx and Rx antenna arrays. An in-house LabView application is used to remotely control the measurement set-up and data logging. The system enables to sequentially retrieve up to 16 channel responses (corresponding to every combination of Tx and Rx array elements, up to four elements in each array). Total acquisition time is of the order of 30 s.

An indoor laboratory was chosen as the measurement environment, Fig. 10. The Rx array was kept stationary at a fixed position, while the Tx array scanned a $1 \mathrm{~m} \times 1 \mathrm{~m}$ area with 10 cm mesh size, yielding $N_{p}=101$ measurement locations. Distance between Rx and Tx antennas was in the order of $4 \mathrm{~m}$. The channel frequency responses corresponding to each of the 16 $\mathrm{Rx} / \mathrm{Tx}$ array element combinations were measured in the 2.5 to $2.7 \mathrm{GHz}$ interval (WiMax and LTE bands) taking $N_{f}=201$ frequency samples.

To force non-line-of-sight link (NLOS) conditions, a metallic cabinet was used to block the direct ray path. It is well known that MIMO performance improvement is best evidenced for NLOS scenarios, where diversity gain is the highest, corresponding to most uncorrelated transmission channels [2]. In order to enhance multipath propagation, some artificial scatters were placed in the room, made from objects covered with ruffled aluminum foil.

Antenna coordinates and orientation, as well as cables, calibration procedures and scenario were exactly the same for both the CXETS array and the patch array. The environment was kept 

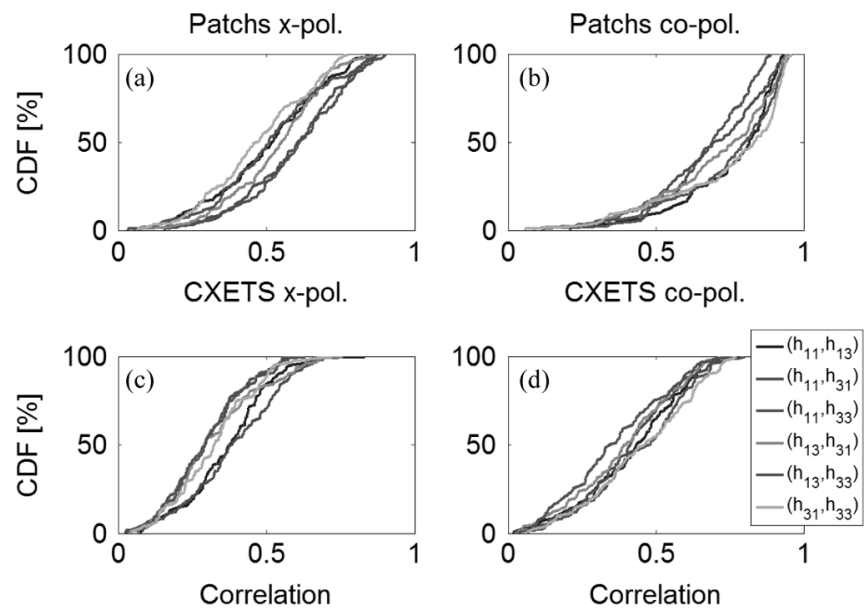

Fig. 11. Measured correlation between channels in a $2 \times 2$ MIMO setup using: (a) two cross-polarized patches (\#1 and \#2); (b) two co-polarized patches (\#1 and \#3); (c) two cross-polarized CXETS (\#1 and \#2); (d) two co-polarized CXETS (\#1 and \#3).

rigorously stationary throughout the whole measurement campaign, including the use of remote data logging to avoid operator influence on the scenario.

Measurements were performed with equal Tx and Rx antennas, instead of using a typical antenna at the personal terminal, because this allows isolating the contribution from the proposed CXETS antenna, without being limited or degraded by the characteristics of the personal terminal antenna.

\section{B. Capacity Estimation}

A first analysis of the measurements is performed using data from only two elements of the array, as in a $2 \times 2$ MIMO configuration $\left(N_{t}=2\right.$ and $\left.N_{r}=2\right)$. Either two cross-polarized array elements in both Tx and Rx are selected (data from antennas \#1 and \#2), or two co-polarized elements are selected (data from antennas \#1 and \#3). This analysis allows confirming the benefit from using polarization diversity and to evaluate how the CXETS and the patch arrays compare with respect to channel capacity in view of their different coupling characteristic.

Maximum MIMO performance requires parallel uncorrelated channels [16]. For the $2 \times 2$ configuration there are 4 parallel channels and therefore, 6 correlation coefficients $\rho$ can be determined between them

$$
\rho=\langle a, b\rangle=\left|\frac{E\left([a-E(a)][b-E(b)]^{*}\right)}{\sqrt{E\left(a^{2}\right)-E(a)^{2}} \sqrt{E\left(b^{2}\right)-E(b)^{2}}}\right|
$$

where $E(\cdot)$ corresponds to the expected value calculated over the measured bandwidth, [.]* represents the complex conjugate, and $a$ and $b$ represent measured channel responses between any two antenna pairs. All six correlation coefficients are determined for each of the $N_{p}=101$ measured positions inside the room. The corresponding cumulative distribution function (CDF) is represented in Fig. 11 for the CXETS array link and for the patch array link, considering either the crossor co-polarized antennas. Therefore, the plot vertical axis indicates the probability of finding a position in the room with channel correlation coefficient below the abscissa value.
The first observation is that for both polarization configurations, correlation is much lower for the CXETS, owing to its better inter-element isolation and polarization purity. Anyway, for both array types, the correlation coefficient is lower when cross-polarized antenna elements are used. This confirms that diversity gain increases when orthogonal polarized antennas are used [4]. It is noted that the decrease in channel correlation is achieved despite the co-polarized antennas being farther from each other as they are laid along the diagonal of the array and not adjacent to each other as with the cross-polarized ones, see Figs. 1 and 5.

Consider the case where only the receiver has channel information; the maximum achievable channel capacity $C$ is given by Shannon's equation [16]

$$
C=\log _{2}\left(I_{N_{r}}+\frac{S N R}{N_{t}} \mathbf{H}_{\mathbf{n}} \mathbf{H}_{\mathbf{n}}^{H}\right) \mathrm{bit} / \mathrm{s} / \mathrm{Hz}
$$

where $I$ is a $N_{r} \times N_{r}$ identity matrix, SNR is the signal-to-noise ratio at the receiver and $[.]^{H}$ represents the Hermitian operator. To exclude the effect of path loss variation on the received power, $\mathbf{H}_{\mathbf{n}}$ is normalized so that the average power is unitary [10], [17], [18]

$$
\mathbf{H}_{\mathbf{n}}=\frac{\mathbf{H}}{\sqrt{\frac{1}{N_{r} N_{t} N_{f} N_{p}} \sum_{N_{r}} \sum_{N_{t}} \sum_{N_{f}} \sum_{N_{p}}|\mathbf{H}|^{2}}} .
$$

Although this normalization removes path loss, it does not hide the effect of propagation-induced correlation or array element coupling-induced correlation. As previously mentioned, the non-normalized channel transfer matrix $\mathbf{H}$ corresponds directly to the S-matrix measured at the Tx and Rx array ports, using a vector network analyzer [17].

Capacity values are calculated for the CXETS and patch arrays at $\mathrm{SNR}=10 \mathrm{~dB}$ for each frequency and position sample in different MIMO configurations, using (2) and the measured channel matrix. Fig. 12 presents the corresponding cumulative distribution function. For reference, Fig. 12 also shows for each MIMO configuration the calculated capacity for a Rayleigh fading channel, that is, each element $h_{n}$ of the normalized channel transfer matrix $\mathbf{H}_{\mathbf{n}}$ is given by

$$
h_{n}=\frac{1}{\sqrt{2}}[\operatorname{Normal}(0,1)+j \operatorname{Normal}(0,1)] .
$$

where $\operatorname{Normal}(0,1)$ is an independent and identically distributed (i.i.d.) value with Gaussian distribution function, zero mean and unit variance $\sigma_{h}^{2}=1$. This corresponds to totally uncorrelated channels.

Capacity improvement from the CXETS array against the array of patches is more evident for higher order MIMO configurations (Fig. 12(c)-(d)). This is clearer when comparing median capacity values (dashed horizontal line in Fig. 12). The results for each type of array are indicated in Table II along with the ratio between the capacity obtained from measured channel data 

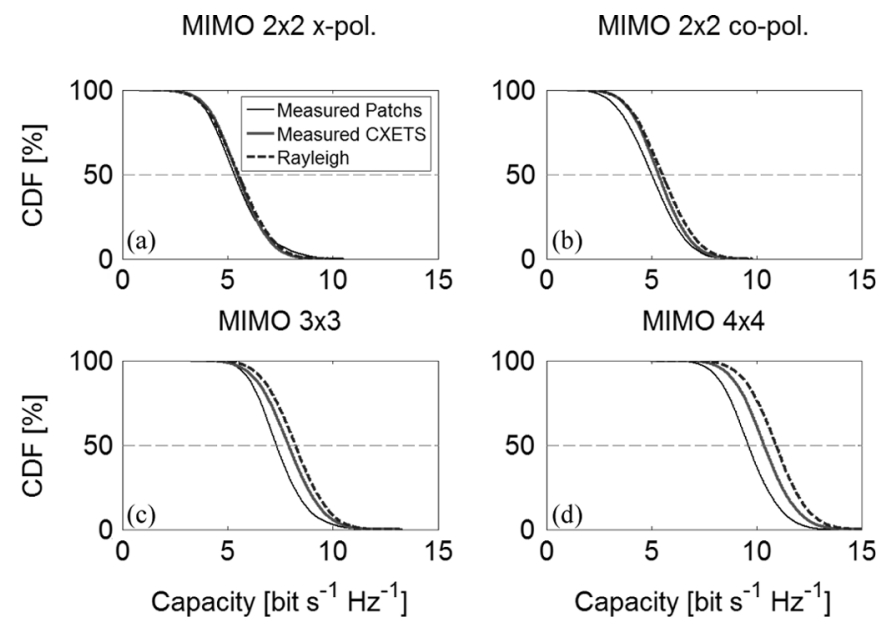

Fig. 12. Measured and simulated capacity for a MIMO set-up with $\mathrm{SNR}=10 \mathrm{~dB}$ at Rx and Tx, assuming power control : (a) two co-polarized array elements (b) two cross-polarized array elements; (c) three array elements; (d) all four array elements.

TABLE II

CAPACITY VALUES FOR 50\% PROBABILITY

\begin{tabular}{|c|c|c|c|c|c|}
\hline \multirow{2}{*}{$\#$} & & \multicolumn{2}{c|}{ Patchs } & \multicolumn{2}{c|}{ CXETS } \\
\cline { 3 - 6 } & & $\begin{array}{c}\text { Capacity } \\
{[\text { bit/s/Hz] }}\end{array}$ & $\begin{array}{c}\text { C } / \\
\mathrm{C}_{\text {Rayleigh }}\end{array}$ & $\begin{array}{c}\text { Capacity } \\
{[\text { bit/s/Hz }]}\end{array}$ & $\begin{array}{c}\mathrm{C} / \\
\mathrm{C}_{\text {Rayleigh }}\end{array}$ \\
\hline a & $2 \times 2 \times$ x-pol. & 5.33 & $96.65 \%$ & 5.48 & $99.46 \%$ \\
\hline b & $2 \times 2$ co-pol. & 5.01 & $90.93 \%$ & 5.34 & $96.96 \%$ \\
\hline c & $3 \times 3$ & 7.32 & $89.15 \%$ & 7.90 & $96.19 \%$ \\
\hline d & $4 \times 4$ & 9.62 & $87.78 \%$ & 10.36 & $94.60 \%$ \\
\hline
\end{tabular}

and the capacity calculated for completely independent channels (Rayleigh curves).

Table II shows that due to antenna mutual coupling and channel correlation, capacity does not scale linearly with number of channels as would be expected if they were independent [16]. In fact, the detrimental effect of mutual coupling on capacity increases with the number of array elements [15]. However it is clear in all cases the importance of better isolation and polarization purity of the CXETS.

These results assume that the system uses power control to fix SNR at constant $10 \mathrm{~dB}$ level. Otherwise, in environments with poor multipath richness, configuration \#b will tend to receive in average more power than configuration \#a. If we want to alternatively compare capacity for constant $T x$ power, we can consider for all cases of Table II the complete measured channel transfer matrix $\mathbf{H}\left(N_{r}=N_{t}=4\right)$ in the normalization (3) and extract afterwards the sub-matrixes from $\mathbf{H}_{\mathbf{n}}$ corresponding to each configuration [18]. For configuration \#a, this leads instead to $4.57 \mathrm{bit} / \mathrm{s} / \mathrm{Hz}$ for the patch array and $5.18 \mathrm{bit} / \mathrm{s} / \mathrm{Hz}$ for the CXETS array, which is not very different from the fixed SNR case thanks to the richness of the tested scenario. As could be expected, the other array configurations capacity remains practically unchanged.

For spatial multiplexed MIMO systems, it is relevant to further evaluate the eigenvalues of the measured $\mathbf{H}_{\mathbf{n}}$. Each eigenvalue represents an orthogonal parallel channel where independent data stream can be transmitted simultaneously, thus increasing the bit rate of the system [2]. Calculations were made
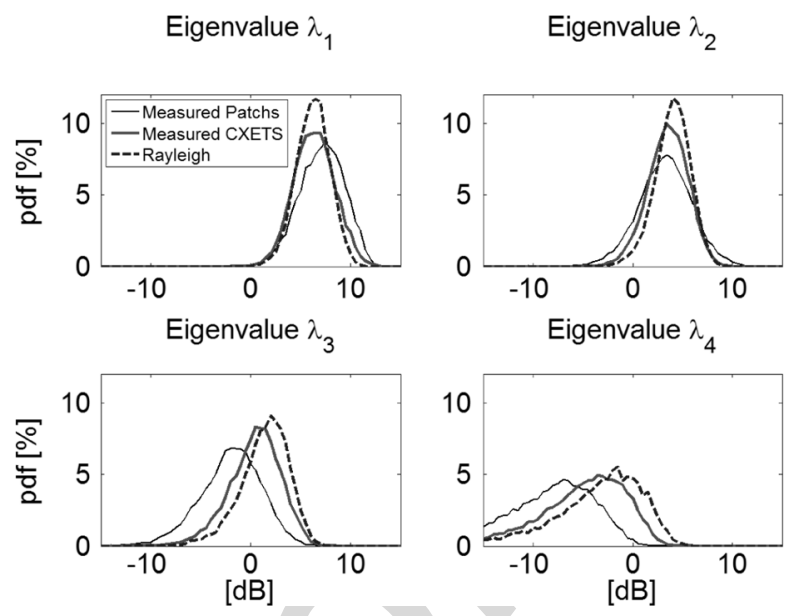

Fig. 13. Measured and simulated eigenvalues of the $4 \times 4$ MIMO setup.

for the $4 \times 4$ MIMO configuration $\left(N_{t}=4\right.$ and $\left.N_{r}=4\right)$, using all $\mathbf{H}_{\mathbf{n}}$ transfer functions measured at each frequency and at each array location in the room. For each measurement, the eigenvalues were ordered such that $\lambda_{1}>\lambda_{2}>\lambda_{3}>\lambda_{4}$. Fig. 13 presents the corresponding probability density functions considering $0.5 \mathrm{~dB}$ eigenvalue amplitude steps (bins).

As the level of correlation increases from the ideal Rayleigh channel case to the measured CXETS and patch array cases, the mean value of the first eigenvalue $\lambda_{1}$ increases while the mean of the other eigenvalues decreases. Such effect reflects the degradation of spatial multiplexed MIMO performance and the tendency to approximate the case of a single independent information channel, as in a SISO configuration. Again the CXETS array performance is much closer to the uncorrelated channel case (Rayleigh curves) than the array of patches due to much lower mutual coupling.

Shannon's (2) determines the maximum achievable capacity, but the actual throughput in a real system depends on further aspects like modulation and coding. A system emulator developed in Matlabltextregistered was used to evaluate the simultaneous transmission of four independent and parallel bit streams using the measured channel transfer matrix in $4 \times 4$ MIMO configuration $\left(N_{\mathrm{t}}=4\right.$ and $\left.N_{r}=4\right)$. For instance using binary phase shift keying (BPSK) modulation and maximum-likelihood (ML) detection at the receiver, the transmission between two CXETS arrays presents a bit error rate (BER) at SNR $=10 \mathrm{~dB}$ that is almost 15 times lower than the equivalent configuration using two arrays of patches.

\section{CONCLUSION}

The proposed CXETS antenna array, with its very low coupling between the closely packed array elements and with its pure linear polarization characteristics, is shown to clearly enhance the known benefits from using space and polarization diversity in MIMO systems, when compared to commonly used antennas, like the array of patches. The large operating bandwidth from 2.4 to $4.8 \mathrm{GHz}$ with stable radiation characteristics allows using the CXETS array for multisystem access points covering services like WiFi, WiMax, LTE and UWB. The antenna cavity back increases the front to back radiation level 
above $12 \mathrm{~dB}$ allowing its mounting on metal surfaces or against a wall for WLAN access points. These cumulative characteristics make CXETS a unique and effective candidate for a very wideband MIMO array.

In order to test its advantage for MIMO applications, basic MIMO performance comparison was conducted at $2.6 \mathrm{GHz}$ (WiMax or LTE bands) between the CXETS array and a narrowband patch array with the same spatial and polarization configuration. Based upon measured channel transfer matrix using a MIMO channel sounder, it was shown that the new array increases channel capacity, better approaching the ideal limit for completely uncorrelated channels.

Although all MIMO performance tests were conducted only at the lower fraction of the CXETS available bandwidth, it is expected that the overall performance will improve further for the rest of the band. In fact, while the antenna radiation characteristics remain almost unchanged across the whole band, for higher frequencies the separation between CXETS array elements becomes larger in terms of the operating wavelength and, therefore, the channels between transmit and receive antennas are expected to become more uncorrelated.

\section{ACKNOWLEDGMENT}

The authors acknowledge the collaboration from V. Fred and C. Brito for prototype construction, and A. Almeida for prototype measurements.

\section{REFERENCES}

[1] S. M. Alamouti, "A simple transmit diversity technique for wireless communications," IEEE J. Sel. Areas Commun., vol. 16, pp. 1451-1458I, Oct. 1998.

[2] D. Tse and P. Viswanath, Fundamentals of Wireless Communication. Cambridge, U.K.: Cambridge Univ. Press, 2005.

[3] R. Janaswamy, "Effect of element mutual coupling on the capacity of fixed length linear arrays," IEEE Antennas Wireless Propag. Lett., vol. 1, pp. 157-160, 2002.

[4] J. Valenzuela-Valdés, M. García-Fernández, A. Martínez-González, and D. Sánchez-Hernández, "The role of polarization diversity for MIMO systems under Rayleigh-fading environments,' IEEE Antennas Wireless Propag. Lett., vol. 5, pp. 534-536, 2006.

[5] J. Costa, C. Medeiros, and C. Fernandes, "Performance of a crossed exponentially tapered slot antenna for UWB systems," IEEE Trans. Antennas Propag., vol. 57, pp. 1345-1352, May 2009.

[6] C. Medeiros, J. Costa, and C. Fernandes, "Compact tapered slot UWB antenna with WLAN band rejection," IEEE Antennas Wireless Propag. Lett., vol. 8, pp. 661-664, 2009.

[7] C. Medeiros, E. Lima, J. Costa, and C. Fernandes, "Wideband slot antenna for WLAN access points," IEEE Antennas Wireless Propag. Lett., vol. 9, pp. 79-82, 2010.

[8] R. Bhatti, J. Choi, and S. Park, "Quad-band MIMO antenna array for portable wireless communications terminals," IEEE Antennas Wireless Propag. Lett., vol. 8, pp. 129-132, 2009.

[9] H. Li, J. Xiong, and S. He, "Extremely compact dual-band PIFAs for MIMO application," Electronics Lett., vol. 45, pp. 869-870, Aug. 2009.

[10] A. Rajagopalan, G. Gupta, A. Konanur, B. Hughes, and G. Lazzi, "Increasing channel capacity of an ultrawideband MIMO system using vector antennas," IEEE Trans. Antennas Propag., vol. 55, pp. 2880-2887, Oct. 2007.

[11] S. Zhang, Z. Ying, J. Xiong, and S. He, "Ultrawideband MIMO/diversity antennas with a tree-like structure to enhance wideband isolation," IEEE Antennas Wireless Propag. Lett., vol. 8, pp. 1279-1282, 2009.
[12] S. Zhang, P. Zetterberg, and S. He, "Printed MIMO antenna system of four closely-spaced elements with large bandwidth and high isolation," Electronics Lett., vol. 46, pp. 1052-1053, July 2010.

[13] M. Manteghi and Y. Rahmat-Samii, "Multiport characteristics of a wide-band cavity backed annular patch antenna for multipolarization operations," IEEE Trans. Antennas Propag., vol. 53, pp. 466-474, Jan. 2005.

[14] [Online]. Available: www.cst.comCST [Online]. Available:

[15] D. Browne, M. Manteghi, M. Fitz, and Y. Rahmat-Samii, "Experiments with compact antenna arrays for MIMO radio communications," IEEE Trans. Antennas Propag., vol. 54, pp. 3239-3250, Nov. 2006.

[16] G. Foschini and M. Gans, "On limits of wireless communications in a fading environment when using multiple antennas," Wireless Personal Commun., vol. 6, pp. 311-335, 1998.

[17] M. Herdin, H. Ozcelik, H. Hofstetter, and E. Bonek, "Variation of measured indoor MIMO capacity with receive direction and position at 5.2 GHz," Electron. Lett., vol. 38, pp. 1283-1285, Oct. 2002.

[18] J. Wallace, M. Jensen, A. Swindlehurst, and B. Jeffs, "Experimental characterization of the MIMO wireless channel: Data acquisition and analysis," IEEE Trans. Wireless Commun., vol. 2, pp. 335-343, Mar. 2003.

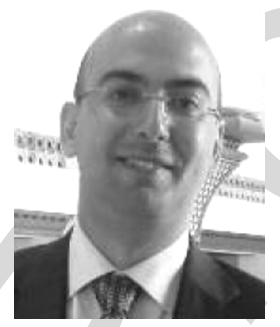

Jorge R. Costa (S'97-M'03-SM'09) was born in Lisbon, Portugal, in 1974. He received the Licenciado and Ph.D. degrees in electrical and computer engineering from the Instituto Superior Técnico (IST), Technical University of Lisbon, Lisbon, Portugal, in 1997 and 2002, respectively.

$\mathrm{He}$ is currently a Researcher at the Instituto de Telecomunicações, Lisbon, Portugal. He is also an Assistant Professor at the Departamento de Ciências e Tecnologias da Informação, Instituto Superior de Ciências do Trabalho e da Empresa. His present research interests include lenses, reconfigurable antennas, MEMS switches, UWB, MIMO and RFID antennas. He is the coauthor of four patent applications and more than 50 contributions to peer reviewed journals and international conference proceedings. More than ten of these papers have appeared in IEEE Journals.

Prof. Costa is currently serving as an Associate Editor for the IEEE TRANSACTIONS ON ANTENNAS AND PROPAGATION.

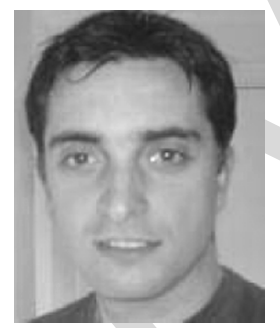

Eduardo B. Lima was born in Viseu, Portugal, in 1978. He received the Licenciado and M.Sc. degrees in electrical and computer engineering from the Instituto Superior Técnico (IST), Lisbon, Portugal, in 2003 and 2008, respectively.

$\mathrm{He}$ is a Researcher and also the software developer for antenna measurements control at the Instituto de Telecomunicações, Lisbon, Portugal. From 2004 to 2007, he was involved on the ESA/ESTEC project ILASH (Integrated Lens Antenna Shaping). He is the coauthor of one patent application and twelve technical papers in international journals and conference proceedings in the area of antennas. His present research interests include dielectric lens antennas and MIMO.

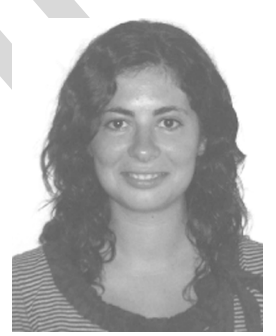

Carla R. Medeiros was born in Ponta Delgada, Açores, Portugal, in 1982. She received the Licenciado and M.Sc. degrees in electrical and computer engineering from the Instituto Superior Técnico (IST), Technical University of Lisbon, Lisbon, Portugal, in 2006 and 2007, respectively.

Since 2006, she has been a Researcher at the Instituto de Telecomunicações (IT), focusing her work on antenna for wireless communications. She collaborates in national research projects. Her current research interests are in the areas of reconfigurable, RFID, MIMO and UWB antennas. 


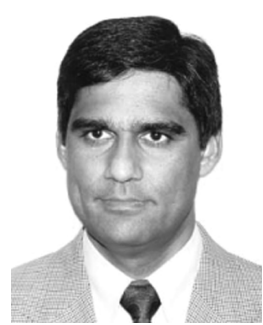

Carlos A. Fernandes (S'86-M'89-SM'08) received the Licenciado, M.Sc., and Ph.D. degrees in electrical and computer engineering from Instituto Superior Técnico (IST), Technical University of Lisbon, Lisbon, Portugal, in 1980, 1985, and 1990, respectively.

He joined the IST in 1980, where he is presently Full Professor at the Department of Electrical and Computer Engineering in the areas of microwaves, radio wave propagation and antennas. He is a Senior Researcher at the Instituto de Telecomunicações and member of the Board of Directors. He has been the Leader of antenna activities in National and European Projects as RACE 2067-MBS (Mobile Broadband System), ACTS AC230-SAMBA (System for Advanced Mobile Broadband Applications) and ESA/ESTEC-ILASH (Integrated Lens Antenna Shaping). He has coauthored a book, a book chapter, and more than 120 technical papers in peer reviewed international journals and conference proceedings, in the areas of antennas and radiowave propagation modeling. His current research interests include dielectric antennas for millimeter wave applications, antennas and propagation modeling for personal communication systems, RFID antennas, artificial dielectrics and metamaterials. 